TITLE:

\title{
Multiobjective robust shape and topology optimization of plane frames using order statistics
}

$\operatorname{AUTHOR}(\mathrm{S}):$

Shen, Wei; Ohsaki, Makoto; Yamakawa, Makoto

\section{CITATION:}

Shen, Wei ...[et al]. Multiobjective robust shape and topology optimization of plane frames using order statistics. Structural and Multidisciplinary Optimization 2021, 63(1): $75-$ 94

\section{ISSUE DATE:}

2021-01

URL:

http://hdl.handle.net/2433/267901

\section{RIGHT:}

This version of the article has been accepted for publication, after peer review (when applicable) and is subject to Springer Nature's AM terms of use, but is not the Version of Record and does not reflect post-acceptance

improvements, or any corrections. The Version of Record is available online at: http://dx.doi.org/10.1007/s00158-02002663-8; The full-text file will be made open to the public on 16 July 2021 in accordance with publisher's 'Terms and Conditions for Self-Archiving'; This is not the published version. Please cite only the published version. この論文は出版 社版でありません。引用の際には出版社版をご確認じ利用ください。 


\title{
Submitted to Structural and Multidisciplinary Optimization
}

\section{Multiobjective Robust Shape and Topology Optimization of Plane Frames using Order Statistics}

\author{
Wei Shen ${ }^{1}$, Makoto Ohsaki ${ }^{1}$, Makoto Yamakawa ${ }^{2}$ \\ ${ }^{1}$ Department of Architecture and Architectural Engineering, Kyoto University, \\ Kyoto-Daigaku Katsura, Nishikyo, Kyoto 615-8540, Japan \\ ${ }^{2}$ Department of Architecture, Tokyo University of Science, Tokyo 125-8585, Japan
}

\begin{abstract}
This paper presents a worst-case approach to robust optimization of plane frame structures under variation in uncertain parameters. The optimization procedure is first implemented without considering uncertainty, resulting in an optimal structure that may be unstable without bending stiffness. Based on such optimal solution we then take variation in uncertain parameters into consideration and estimate the quantile response or trimmed mean of order statistics, where the quantile response is used as a relaxation of worst value of structural response. In order to obtain robust optimal solutions at various robustness levels, a multiobjective optimization problem is formulated and solved to simultaneously minimize the several order statistics or trimmed means with different orders. It is demonstrated in the numerical examples that the optimal distribution of cross-sectional areas of elements vary with the change of robustness level, and the convergence by using trimmed mean as estimation of quantile response is better than that of the simple order statistics.
\end{abstract}

Keywords: Plane frame structure; Multiobjective robust optimization; Order statistics; Genetic algorithm;

\section{Introduction}

Traditional optimization of truss-like structure has been constantly receiving attention since the pioneering work by Maxwell (1870) and Michell (1904) with deterministic structural and loading parameters. However, the resulting optimal solution may be vulnerable to the uncertainty which is inevitable for real-world structures. Therefore, it is more reasonable to consider the uncertain characteristics of parameters during the optimization process and explore the effect of randomness on structural design. There have been extensive researches and studies that take the uncertain parameters into account during the recent 30 years (Choi et al. 2007; Ben-Tal et al. 2009; Elishakoff and Ohsaki 2010), in which the methods are based on, roughly speaking, probabilistic model or non-probabilistic model for describing the uncertain characteristics. 
When based on probabilistic model, the uncertain parameters are considered as random variables with known or partially known distribution, and the structural optimization problem is usually formulated as minimizing the structural volume under failure probability constraint, which is sometimes referred to as reliability-based structural optimization (RBSO) (Frangopol 1985), or under the weighted sum of statistical moments of structural response, which is often called robust structural optimization (RSO) (Ben-Tal and Nemirovski 2002). For RBSO (Enevoldsen and Sorensen 1994; Kharmanda et al. 2004; Mogami et al. 2006), many strategies such as the decoupled single-loop algorithms (Du and Chen 2004; Kogiso et al. 2012) and approximation of the limit state function (Qu and Haftka 2004; Cheng et al. 2006) are proposed to improve efficiency of the double-loop algorithms, where the loop of reliability index calculation is nested in the outer loop of structural optimization. However, the computation cost will significantly increase when the system reliability or multiple failure modes are taken into consideration (Lee et al. 2010). On the other hand, RSO aims at finding the designs that are less sensitive to the uncertain parameters. Variability of the structural response under uncertainty is often represented by its second-order statistical moment, and is simultaneously minimized or assigned as constraints with its first-order statistical moment (i.e., mean value) (Lee and Park 2001; Dunning and Kim 2013). However, substantial efforts are needed for the calculation of statistical moments and their derivatives if more than one type of random variables are involved, or uncertainty is assumed in the structural stiffness because the displacements is a function of the inverse of stochastic stiffness matrix (Guest and Igusa 2008; Asadpoure et al. 2011). Recently, stochastic method has been introduced in both RBSO and RSO problems to describe the characteristics of the uncertain parameters, and it attracts more attention from the researchers due to its explicit form of uncertainty propagation (Doltsinis and Kang 2004; Tootkaboni et al. 2012; Jalalpour et al. 2013). However, balancing the accuracy and efficiency is still a challenge and such kind of method has not been fully explored yet. In addition, both RBSO and RSO require accurate distribution types of the uncertain parameters to ensure the accuracy of the solution, and a large estimation error will occur when the information about uncertainty is insufficient (Du and Choi 2008; Picheny et al. 2010). Wang et al $(2018,2019)$ developed sequential and concurrent multiscale reliability-based optimization methods for continuum structures with unknown-but-bounded uncertainties, and a novel nonprobabilistic reliability measure is derived by using interval mathematics. The scale-span uncertainty qualification is exploited between macro topology optimization and micro sizing optimization. However, since the interval paramedic vertex theorem is adopted, the scale-span uncertainty qualification is available when monotonicity hypothesis and requirements of finite uncertainty dimensions is satisfied, and the efficiency and accuracy may be influenced by the nonlinearity and convexity of uncertainty.

By contrast, the non-probabilistic model provides an alternative if the distribution types of uncertain parameters are not known in advance, and the structural optimization under such uncertainty framework, called worst-case optimization (WCO) (Ben-Tal et al. 2009), generally minimizes the structural response with the worst combination of uncertain parameters in the predefined set. Traditionally, the procedure of WCO consists of a two-level problem: the upperand lower-level problems are solved for structural optimization and worst-case evaluation, respectively (Du and Chen 2000; Au et al. 2003; Takezawa et al. 2011). However, the computation cost for searching the exact worst value is very large even for a simple interval model or a well- 
defined ellipsoid model (Lombardi and Haftka 1998; Ohsaki and Katsura 2012). To save computation cost without loss of generality, Kanno and Takewaki (2006a,b) constructed the minimum confidence ellipsoid of the structural response to solve the WCO problem in a confidence way; Guo et al. (2009) extended this method to a single level formulation under stiffness uncertainty. Note that they mainly concern single-objective optimization problems. However, it will be useful for designers if the structures which minimize the response at different levels of robustness can be obtained. Thus, the problem becomes a multiobjective optimization problem.

Though there exist large number of researches about multiobjective structural optimization, most of them have only two objective functions (Venter and Haftka 2010; Zavala et al. 2014; Izui et al. 2015). Richardson et al. (2015) formulated a multiobjective RSO by selecting different linear combinations of the mean and the standard deviation of compliance as separate objective functions. Ohsaki et al. (2019) recently developed an order statistic approach to consider the worst-case value with certain confidence level, in which the worst-case value is approximated by the $k$ th order statistics and the parameter $k$ is regarded as the robustness level according to the theory of distribution free tolerance interval (Prescott et al. 1993). Based on this idea, we present a new multiobjective formulation for robust topology optimization of frame structures under uncertainty in stiffness and geometry to minimize the structural stress under volume constraint. The order statistics corresponding to various robustness levels related to the worst, quartile and median responses under interval uncertainty as well as the nominal response without uncertainty are considered as objective functions in the multiobjective optimization problem, and the trimmed means are also introduced to enhance the convergence of optimization process. The properties of robust optimal topologies are investigated in comparison to those of trusses. The design variables are the cross-sectional areas and the nodal locations to simultaneously optimize the stiffness, shape and topology of the plane frame. Pareto optimal solutions are found using a multiobjective genetic algorithm.

The rest of this paper is organized as follows. In Section 2 the worst-case value of structural response and its relaxed approximation by order statistics are discussed, while the formulation of multiobjective optimization problem is given in Section 3. Section 4 presents the implementation details about shape and topology optimization of frame structures, which is based on the formulation of multiobjective optimization in Section 3, and three numerical examples are presented in Section 5 to demonstrate the effectiveness of the proposed method. Finally, some conclusions are drawn in Section 6.

\section{Worst-case approximation using order statistics}

There are various types of formulations of RSO, and the most general way is to minimize the structural cost represented by material volume under the constraint on structural response considering uncertainty in parameters. If the worst value is used for the structural response, the problem is also called WCO or worst-case design, for which accurate evaluation of the worst response is necessary. For simplicity, we formulate a problem of minimizing the worst response 
under volume constraint. Hence, we consider an RSO problem of plane frames and assume that uncertainty exists only in the objective function.

Let $\boldsymbol{\theta}=\left(\theta_{1}, \theta_{2}, \ldots \theta_{s}\right) \in \Theta$ denote the vector of $s$ uncertain parameters in the space $\Theta$ and $\overline{\boldsymbol{\theta}}=\left(\bar{\theta}_{1}, \bar{\theta}_{2}, \ldots, \bar{\theta}_{s}\right)$ denote the vector of their nominal values. The vector $\boldsymbol{X}=\left(X_{1}, X_{2}, \ldots X_{n}\right) \in \Omega$ represents the $n$ design variables in the design variable space $\Omega$. Suppose both $\Theta$ and $\Omega$ are continuous and bounded, i.e., they are closed sets.

The structural optimization problem without considering uncertainty can be formulated as

$$
\begin{aligned}
& \text { Minimize } f(\boldsymbol{X} ; \overline{\boldsymbol{\theta}}) \\
& \text { subject to } g_{i}(\boldsymbol{X}) \leq 0, \quad i=1,2, \ldots, n_{\mathrm{g}} ; \quad \boldsymbol{X} \in \Omega
\end{aligned}
$$

where the variables are only $\boldsymbol{X}$, and $f(\boldsymbol{X} ; \overline{\boldsymbol{\theta}})$ represents the structural response at nominal value of uncertain parameters. $g_{i}(\boldsymbol{X} ; \overline{\boldsymbol{\theta}})$ is the ith constraint function and $n_{\mathrm{g}}$ is the number of constraints. After solving problem (1), the topology of the structure is modified by removing the elements which have small cross-sectional areas and are considered to have little contribution to the reduction of structural response. However, such unnecessary elements may become useful for a solution to an RSO problem to minimize the worst structural response for specified variation in uncertain parameters, which can be written as

$$
\begin{aligned}
& \text { Minimize } f^{\max }(\boldsymbol{X})=\max _{\boldsymbol{\theta} \in \Theta} f(\boldsymbol{X} ; \boldsymbol{\theta}) \\
& \text { subject to } g_{i}(\boldsymbol{X}) \leq 0, \quad i=1,2, \ldots, n_{\mathrm{g}} ; \quad \boldsymbol{X} \in \Omega
\end{aligned}
$$

where $f^{\max }(\boldsymbol{X})$ represents the maximum structural response within the parameter space $\Theta$. However, directly solving problem (2) is computationally expensive due to the search of $f^{\max }(\boldsymbol{X})$ at each iteration of the optimization procedure. To reduce the computation effort for finding the worst value, the $100 \beta$ th $(0<\beta<1)$ quantile response is used as a relaxation representation of the worst structural response. For this purpose, order statistics is introduced as a quantile estimator.

Let $\boldsymbol{\theta}_{1}, \boldsymbol{\theta}_{2}, \ldots, \boldsymbol{\theta}_{m}$ denote $m$ independent and identically distributed (i.i.d.) vectors of uncertain parameters sampled from $\Theta$. The corresponding structural responses with a specified design variable $\boldsymbol{X}=\left(X_{1}, X_{2}, \ldots X_{n}\right)$ are denoted by $Y_{1}=f\left(\boldsymbol{X}, \boldsymbol{\theta}_{1}\right), Y_{2}=f\left(\boldsymbol{X}, \boldsymbol{\theta}_{2}\right), \ldots, Y_{m}=f\left(\boldsymbol{X}, \boldsymbol{\theta}_{m}\right)$. Because $Y_{1}, Y_{2}, \ldots, Y_{m}$ are i.i.d. uncertain parameters and derived from the same response function, it is rational to assume that these responses are samples from the same distribution that has the cumulative distribution function (cdf) denoted by $F(y)=\operatorname{Pr}\{Y \leq y\}$. We further define the values $Y_{1: m}, Y_{2: m}, \ldots, Y_{m: m}$ to be a permutation of $Y_{1}, Y_{2}, \ldots, Y_{m}$ such that $Y_{1: m} \geq Y_{2: m} \geq \cdots \geq Y_{m: m}$, and the $k$ th $(1 \leq k \leq m)$ response $Y_{k: m}$ is called the $k$ th order statistics. Note that the descending order statistics herein is used for convenience to approximate the maximum structural response, which is different from the conventional definition of order statistics that is arranged in an ascending order. 
Based on the statistical inference theory in order statistics (Prescott et al. 1993), the probability $\alpha_{k}$ of $100 \beta \%$ of the structural response $f(\boldsymbol{X} ; \boldsymbol{\theta})$ less than the $k$ th order statistics $Y_{k: m}$ can be calculated as

$$
\alpha_{k}=\operatorname{Pr}\left\{F\left(Y_{k: m}\right) \geq \beta\right\}=\operatorname{Pr}\left\{U_{k: m} \geq \beta\right\}=\sum_{r=0}^{m-k}\left(\begin{array}{c}
m \\
r
\end{array}\right) \beta^{r}(1-\beta)^{m-r}=1-I_{\beta}(m-k+1, k)
$$

where $U_{k: m}$ is the $k$ th order statistics from $m$ i.i.d. uniform random variables in the range [0,1], and $I_{\beta}(m-k+1, k)$ is the incomplete beta function (Prescott et al. 1993; Ohsaki et al. 2019). Equation (3) is also called distribution-free one-side tolerance interval because the probability of the structural response falling into the one-side interval $\left(-\infty, Y_{k: m}\right)$ is independent of the distribution of $Y_{k: m}$, and $Y_{k: m}$ can be seen as the $100 \beta \%$ quantile response in probabilistic sense.

In order to enable $Y_{k: m}$ to be accurate as a quantile estimator of the structural response, the value of the probability $\alpha_{k}$ in Eq. (3) should be close to 1; e.g., 0.9 or 0.95 . Furthermore, according to Prescott et al. (1993), $\alpha_{k}$ will monotonically increase to 1 as the sample size $m$ is increased. Therefore, if the values of $k, \alpha_{k}$ and $\beta$ are specified, one can obtain the threshold (minimum) value of $m$ such that $\alpha_{k}$ is not less than its preassigned value; on the other hand, if $m$ is specified as the threshold value satisfying Eq. (3) and the value of $k$ is also given, the relation between $\alpha_{k}$ and $\beta$ can also be derived by solving Eq. (3). As demonstrated by Ohsaki et al. (2019), the relation between $\alpha_{k}$ and $\beta$ can be obtained for some fixed values of $m$ and various specified values of $k(=1,2, \ldots 20)$, which is shown in Fig. 1 for completeness of the paper. Note that the curves in top-right and bottom-left in each figure in Fig. 1 correspond to $k=1$ and 20, respectively. The relations between $k$ and $\beta$ with $\alpha_{k}=0.9$ are also given in Tables 1 and 2 for $m$ $=100$ and 200, respectively. It can be seen from these tables that $\beta$ is a decreasing function of $k$, in other words, the portion of the structural response less than $Y_{k: m}$ will decrease as the value of $k$ increases with the same probability $\alpha_{k}$. Therefore, for given $\alpha_{k}$ and $m$, a higher order $k$ corresponds to a less structural response under uncertainty exceeding $100 \beta$ th quantile response, and the order $k$ and its corresponding order statistics $Y_{k: m}$ approximating the quantile response can be regarded as representing the robustness level of the structure.

Table 1 Relation between $k$ and $\beta\left(\alpha_{k}=0.9, m=100\right)$

\begin{tabular}{|c|c|c|c|c|c|c|c|c|c|c|}
\hline$k$ & 1 & 2 & 3 & 4 & 5 & 6 & 7 & 8 & 9 & 10 \\
\hline$\beta$ & 0.977 & 0.962 & 0.948 & 0.934 & 0.922 & 0.909 & 0.897 & 0.885 & 0.873 & 0.862 \\
\hline$k$ & 11 & 12 & 13 & 14 & 15 & 16 & 17 & 18 & 19 & 20 \\
\hline$\beta$ & 0.850 & 0.839 & 0.827 & 0.816 & 0.805 & 0.794 & 0.783 & 0.772 & 0.761 & 0.750 \\
\hline
\end{tabular}


Table 2 Relation between $k$ and $\beta\left(\alpha_{k}=0.9, m=200\right)$

\begin{tabular}{|c|c|c|c|c|c|c|c|c|c|c|}
\hline$k$ & 1 & 2 & 3 & 4 & 5 & 6 & 7 & 8 & 9 & 10 \\
\hline$\beta$ & 0.989 & 0.981 & 0.974 & 0.967 & 0.960 & 0.954 & 0.948 & 0.942 & 0.936 & 0.930 \\
\hline$k$ & 11 & 12 & 13 & 14 & 15 & 16 & 17 & 18 & 19 & 20 \\
\hline$\beta$ & 0.924 & 0.918 & 0.912 & 0.907 & 0.901 & 0.895 & 0.890 & 0.884 & 0.878 & 0.873 \\
\hline
\end{tabular}

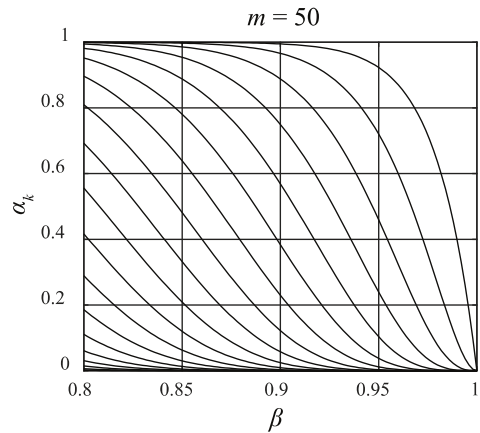

(a)

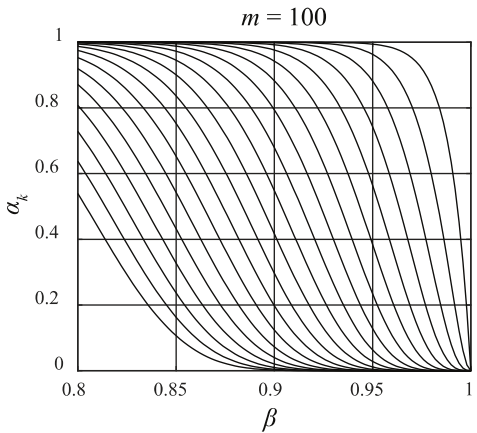

(b)

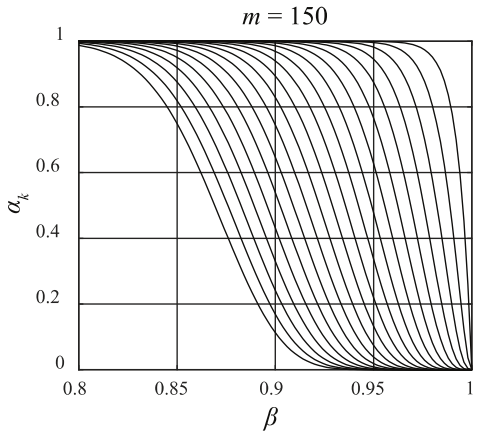

(c)

Fig. 1 Relation between $\alpha_{k}$ and $\beta$ for some fixed values of $m$ and various values of $k$ :

(a) $m=50$; (b) $m=100$; (c) $m=150$

\section{Multiobjective optimization problem}

As discussed in Section 2, the worst structural response under uncertainty can be relaxed to a quantile response, and the robustness level can be represented by the order statistics. Hence, suppose the worst structural response $f^{\max }(\boldsymbol{X})=\max _{\boldsymbol{\theta} \in \Theta} f(\boldsymbol{X} ; \boldsymbol{\theta})$ is approximated by the $k$ th order statistics $Y_{k: m}(\boldsymbol{X} ; \boldsymbol{\theta})$ among $m$ samples, and rewrite problem (2) as

$$
\begin{aligned}
& \text { Minimize } Y_{k: m}(\boldsymbol{X} ; \boldsymbol{\theta}) \\
& \text { subject to } g_{i}(\boldsymbol{X}) \leq 0, \quad i=1,2, \ldots, n_{\mathrm{g}} ; \quad \boldsymbol{X} \in \Omega
\end{aligned}
$$

The estimated worst response approaches the exact extreme value if the value of $k$ is close to 1 . However, the selection of $k$ is not evident, and one may decide the value of $k$ to meet the practical robustness, because the exact extreme value corresponds to extreme rare event. For some cases it may be important to minimize the median and/or percentile values of the response. Therefore, it is of interest to consider the structural response with different values of $k$ in the objective function of

problem (4). The response without considering uncertainty denoted by $\bar{Y}=f(\boldsymbol{X} ; \overline{\boldsymbol{\theta}})$, which is the objective function of problem (1), is also included. Hence, a multiobjective optimization problem is formulated as 


$$
\begin{aligned}
& \text { Minimize } \bar{Y}(\boldsymbol{X} ; \overline{\boldsymbol{\theta}}), Y_{k_{1}: m}(\boldsymbol{X} ; \boldsymbol{\theta}), Y_{k_{2}: m}(\boldsymbol{X} ; \boldsymbol{\theta}), \ldots, Y_{k_{f}: m}(\boldsymbol{X} ; \boldsymbol{\theta}) \\
& \text { subject to } g_{i}(\boldsymbol{X}) \leq 0, \quad i=1,2, \ldots, n_{\dot{\mathrm{g}}} \quad \boldsymbol{X} \in \Omega
\end{aligned}
$$

where $k_{1}, k_{2}, \ldots, k_{f}\left(1 \leq k_{1}<k_{2}<\ldots<k_{f} \leq m\right)$ are the selected $f$ values of order $k$.

According to the dependence properties of order statistics (Boland et al. 1996; Avérous et al. 2005), two order statistics are non-negatively correlated if they are i.i.d. random variables. Since the distribution of structural response is unknown, only distribution-free measurements of dependence, like Spearman's correlation and Kendall's tau can be applied, and their explicit calculation has been exploited by Navarro and Balakrishnan (2010). However, it is known that the value of $Y_{k: m}(\boldsymbol{X} ; \boldsymbol{\theta})$ strongly depends on the choice of random numbers, especially when $k$ is close to 1 . Therefore, in some cases the $L$-statistics, which is a linear combination of order statistics, is also used as an estimator due to its simplicity and stability (Prescott et al. 1993). We use the simple trimmed mean $S_{\bar{k}: m}(\boldsymbol{X} ; \boldsymbol{\theta})$, defined as follows, for smoothing the quantile response:

$$
S_{\bar{k}: m}(\boldsymbol{X} ; \boldsymbol{\theta})=\frac{1}{\left[m p_{2}\right]-\left[m p_{1}\right]} \sum_{k=\left[m p_{1}\right]+1}^{\left[m p_{2}\right]} Y_{k: m}(\boldsymbol{X} ; \boldsymbol{\theta})
$$

where $p_{1}$ and $1-p_{2}\left(0 \leq p_{1}<p_{2} \leq 1\right)$ are the preassigned portions of the samples trimmed at lower and upper ends, and $\left[m p_{1}\right]$ and $\left[m p_{2}\right]$ stand for the greatest integer less than $m p_{1}$ and $m p_{2}$, respectively. The subscript $\bar{k}$ represents the arithmetic mean of all orders $k$ in the set $\left\{\left[m p_{1}\right]+1, \ldots,\left[m p_{2}\right]\right\}$.

It is obvious that if $p_{1}$ and $p_{2}$ in Eq. (6) are properly selected such that

$$
S_{\bar{k}: m}(\boldsymbol{X} ; \boldsymbol{\theta})=\frac{1}{3}\left(Y_{k-1: m}(\boldsymbol{X} ; \boldsymbol{\theta})+Y_{k: m}(\boldsymbol{X} ; \boldsymbol{\theta})+Y_{k+1: m}(\boldsymbol{X} ; \boldsymbol{\theta})\right)
$$

then the trimmed mean $S_{\bar{k}: m}(\boldsymbol{X} ; \boldsymbol{\theta})$ is a function of order $k$, and can also be used as an approximation of quantile response $Y_{k: m}(\boldsymbol{X} ; \boldsymbol{\theta})$ to meet the various robustness levels. We denote the trimmed mean defined as Eq. (7) by $S_{k: m}$ for the implication of function of order $k$ hereafter. Then, the multiobjective optimization problem to minimize $S_{k: m}$ can be formulated as

$$
\begin{aligned}
& \text { Minimize } \bar{Y}(\boldsymbol{X} ; \overline{\boldsymbol{\theta}}), S_{k_{1}: m}(\boldsymbol{X} ; \boldsymbol{\theta}), S_{k_{2}: m}(\boldsymbol{X} ; \boldsymbol{\theta}), \ldots, S_{k_{s}: m}(\boldsymbol{X} ; \boldsymbol{\theta}) \\
& \text { subject to } g_{i}(\boldsymbol{X}) \leq 0, \quad i=1,2, \ldots, n_{\dot{\mathrm{g}}} \quad \boldsymbol{X} \in \Omega
\end{aligned}
$$

where $k_{1}, k_{2}, \ldots k_{s}\left(2 \leq k_{1}<k_{2}<\ldots<k_{s} \leq m-1\right)$ are the selected values of order $k$ in $S_{k: m}(\boldsymbol{X} ; \boldsymbol{\theta})$. 


\section{Robust Shape and Topology Optimization of Plane Frame Structure}

Generally, in shape and topology optimization of plane frame structure, the design variables are the vectors of nodal coordinates $\boldsymbol{x}=\left(x_{1}, x_{2}, \ldots, x_{n_{x}}, y_{1}, y_{2}, \ldots, y_{n_{y}}\right)$ including $x$ - and $y$-coordinates, and cross-sectional areas $\boldsymbol{A}=\left(A_{1}, A_{2}, \ldots, A_{n_{\mathrm{e}}}\right)$, where $n_{x}$ and $n_{y}$ are the numbers of variables in $x$ and $y$-coordinates, respectively, and $n_{\mathrm{e}}$ is the number of elements. The uncertain parameters $\boldsymbol{\theta}$ are assumed to be vectors of Young's modulus of the elements $\boldsymbol{E}_{\mathrm{p}}$ and nodal locations $\boldsymbol{x}_{\mathrm{p}}$. Note that the elements of $\boldsymbol{x}$ and $\boldsymbol{x}_{\mathrm{p}}$ do not exactly correspond with each other; $\boldsymbol{x}$ may have more elements than $\boldsymbol{x}_{\mathrm{p}}$ and vice versa. The nominal values of Young's modulus are denoted by the vector $\overline{\boldsymbol{E}}_{\mathrm{p}}$. Then problem (1) can be specifically written as

$$
\begin{aligned}
& \text { Minimize } f\left(\boldsymbol{A}, \boldsymbol{x} ; \overline{\boldsymbol{E}}_{\mathrm{p}}\right) \\
& \text { subject to } g_{i}(\boldsymbol{A}, \boldsymbol{x}) \leq 0, \quad i=1,2, \ldots, n_{\mathrm{g}} ; x_{\mathrm{L}} \leq x_{j_{1}} \leq x_{\mathrm{U}}, j_{1}=1,2, \ldots, n_{x} \\
& \qquad y_{\mathrm{L}} \leq y_{j_{2}} \leq y_{\mathrm{U}}, j_{2}=1,2, \ldots, n_{y} ; A_{\mathrm{L}} \leq A_{l} \leq A_{\mathrm{U}}, \quad l=1,2, \ldots, n_{\mathrm{e}}
\end{aligned}
$$

where the subscripts $L$ and $U$ represent the lower and upper bounds for the variables, respectively. As discussed in Section 2, some of the elements that have small cross-sectional areas in the optimal solution of problem (9) may contribute to the reduction of structural response when uncertainty is considered. Therefore, based on the optimal shape obtained by solving problem (9), we choose the cross-sectional areas as the design variables for multiobjective optimization problems (5) and (8) which are written, respectively, as follows:

$$
\begin{aligned}
& \text { Minimize } \bar{Y}\left(\boldsymbol{A} ; \overline{\boldsymbol{E}}_{\mathrm{p}}, \overline{\boldsymbol{x}}_{\mathrm{p}}\right), Y_{k_{1}: m}\left(\boldsymbol{A} ; \boldsymbol{E}_{\mathrm{p}}, \boldsymbol{x}_{\mathrm{p}}\right), Y_{k_{2}: m}\left(\boldsymbol{A} ; \boldsymbol{E}_{\mathrm{p}}, \boldsymbol{x}_{\mathrm{p}}\right), \ldots, Y_{k_{f}: m}\left(\boldsymbol{A} ; \boldsymbol{E}_{\mathrm{p}}, \boldsymbol{x}_{\mathrm{p}}\right) \\
& \text { subject to } g_{i}(\boldsymbol{A}, \boldsymbol{x}) \leq 0, \quad i=1,2, \ldots, n_{\mathrm{g}} ; x_{\mathrm{L}} \leq x_{j_{1}} \leq x_{\mathrm{U}}, j_{1}=1,2, \ldots, n_{x} \\
& \qquad y_{\mathrm{L}} \leq y_{j_{2}} \leq y_{\mathrm{U}}, j_{2}=1,2, \ldots, n_{y} ; A_{\mathrm{L}} \leq A_{l} \leq A_{\mathrm{U}}, \quad l=1,2, \ldots, n_{\mathrm{e}} \\
& \text { Minimize } \bar{Y}\left(\boldsymbol{A} ; \overline{\boldsymbol{E}}_{\mathrm{p}}, \overline{\boldsymbol{x}}_{\mathrm{p}}\right), S_{k_{1}: m}\left(\boldsymbol{A} ; \boldsymbol{E}_{\mathrm{p}}, \boldsymbol{x}_{\mathrm{p}}\right), S_{k_{2}: m}\left(\boldsymbol{A} ; \boldsymbol{E}_{\mathrm{p}}, \boldsymbol{x}_{\mathrm{p}}\right), \ldots, S_{k_{s}: m}\left(\boldsymbol{A} ; \boldsymbol{E}_{\mathrm{p}}, \boldsymbol{x}_{\mathrm{p}}\right) \\
& \text { subject to } g_{i}(\boldsymbol{A}, \boldsymbol{x}) \leq 0, \quad i=1,2, \ldots, n_{\mathrm{g}} ; x_{\mathrm{L}} \leq x_{j_{1}} \leq x_{\mathrm{U}}, \quad j_{1}=1,2, \ldots, n_{x} \\
& \qquad y_{\mathrm{L}} \leq y_{j_{2}} \leq y_{\mathrm{U}}, \quad j_{2}=1,2, \ldots, n_{y} ; A_{\mathrm{L}} \leq A_{l} \leq A_{\mathrm{U}}, \quad l=1,2, \ldots, n_{\mathrm{e}}
\end{aligned}
$$

where $\overline{\boldsymbol{x}}_{\mathrm{p}}$ is the nominal values of $\boldsymbol{x}_{\mathrm{p}}$.

After obtaining the Pareto optimal solutions of problems (10) and (11), we remove those elements with small cross-sectional areas to obtain the final topology of the frame.

In the following numerical examples, problems (9), (10) and (11) are solved using genetic algorithms (GAs). It is well known for GAs that the diversity of the initial population has great influence on the final solution and convergence property. Therefore, we use Latin hypercube sampling (LHS) method to generate the initial population for all of the three problems to maintain 
diversity, and add the optimal solution of problem (9) into the initial population of multiobjective optimization problems (10) and (11) for better convergence.

In summary, the robust optimization procedure of plane frame is illustrated in Fig. 2, which consists of the following steps:

Step 1: Select the design variables from the sets of nodal coordinates and cross-sectional areas, and specify the nominal values of uncertain parameters for problem (9). Generate the initial population using LHS method. Solve problem (9) using GA and obtain the optimal solution.

Step 2: Based on the shape of optimal solution of Step 1, select the cross-sectional areas as design variables for multiobjective optimization problems (10) and (11). Generate the initial population using LHS method and add the optimal solution of problem (9) into it.

Step 3: Select the multiple values of order $k(k \leq m)$ to determine the objective functions of problems (10) and (11) according to various robustness levels. Generate $m$ vectors of random values from the specified probability distribution for computing uncertain parameters $\boldsymbol{E}_{\mathrm{p}}$ and $\boldsymbol{x}_{\mathrm{p}}$ in Step 4 to obtain the sample set for order statistics.

Step 4: Solve problems (10) and (11) using a multiobjective GA. For each individual, compute $m$ response values corresponding to the $m$ vectors of $\boldsymbol{E}_{\mathrm{p}}$ and $\boldsymbol{x}_{\mathrm{p}}$, and rearrange the responses in a descending order to compute the objective function values based on the selected orders $k$.

Step 5: Obtain the Pareto optimal solutions, and remove the elements whose cross-sectional areas are small enough. 


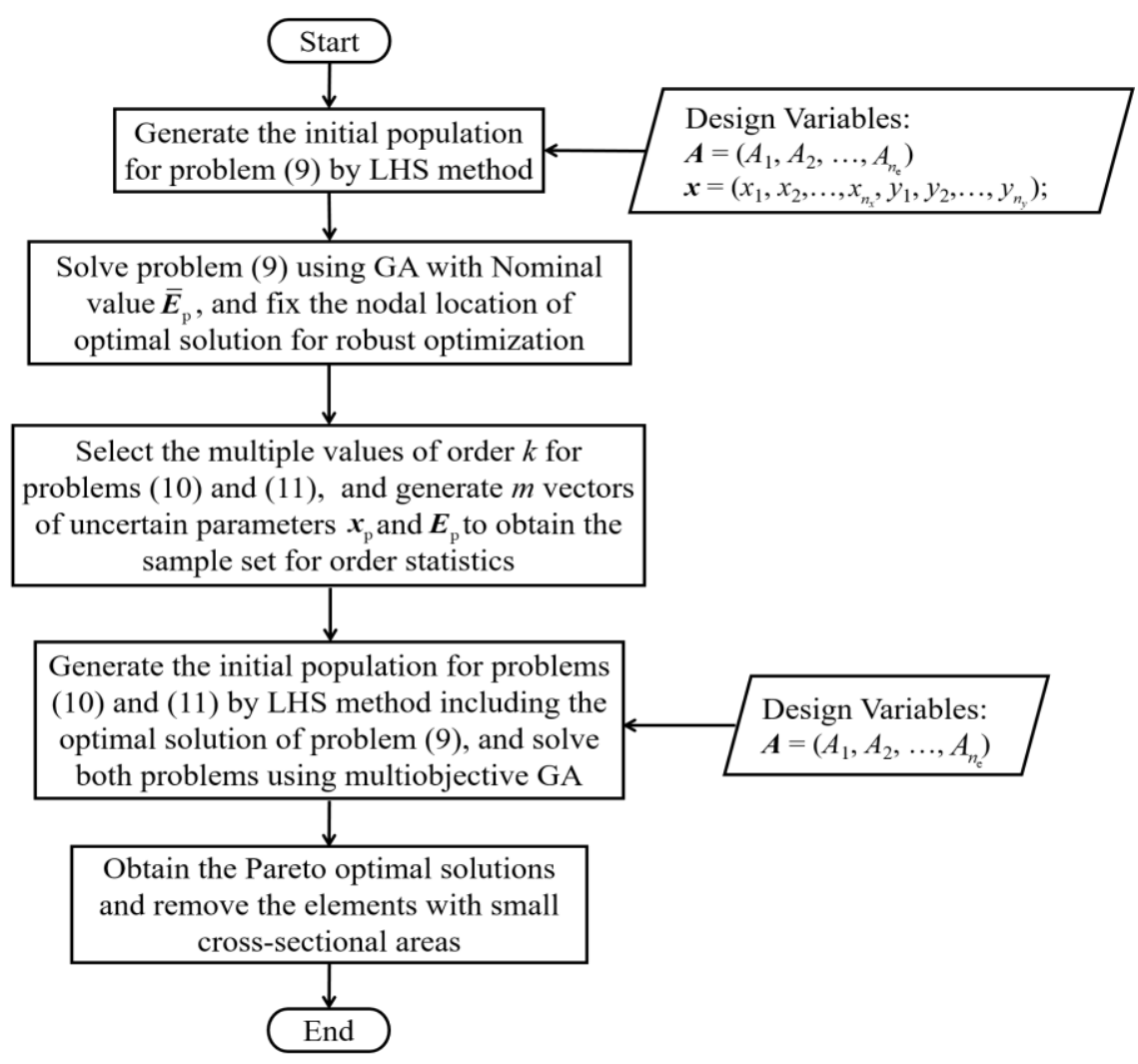

Fig. 2 Flowchart of robust optimization of plane frame structure

\section{Numerical Examples}

In this section, several examples are presented and discussed in order to illustrate the effectiveness of the proposed method. GAs for solving single and multiobjective optimization problems are implemented by using a computer with Core i7 processor and the Global Optimization Toolbox of MATLAB 2018a (Mathworks 2018) with crossover rate 0.7, and the fraction of individuals to keep on the Pareto front is 0.35 by default. The algorithm stops if the average relative change in spread over a consecutive sequence of generations is less than the function tolerance and the final spread is less than the mean spread over the past consecutive sequence of generations. The default values of the length of consecutive sequence and the function tolerance are 100 and $1 \times 10^{-4}$, respectively. Note that the average relative change in spread is calculated by means of geometric average in which the relative change of the $k$ th generation in the consecutive sequence, where $k$ is the number of generations prior to the current generation, has a weighting coefficient $(1 / 2)^{k}$. See MATLAB 2018a (Mathworks 2018) for details.

\subsection{Parameter setting}

We assume that each member has solid circular cross-section, and thereby the element diameter and second moment of inertia can be expressed by its cross-sectional area. In each of the following examples, the uncertain parameters are characterized by the uniformly distributed interval 
variables, i.e., $\boldsymbol{E}_{\mathrm{p}} \in\left[\boldsymbol{E}_{\mathrm{p}, \mathrm{L}}, \boldsymbol{E}_{\mathrm{p}, \mathrm{U}}\right]$ and $\boldsymbol{x}_{\mathrm{p}} \in\left[\boldsymbol{x}_{\mathrm{p}, \mathrm{L}}, \boldsymbol{x}_{\mathrm{p}, \mathrm{U}}\right]$, where $\boldsymbol{E}_{\mathrm{p}, \mathrm{L}}, \boldsymbol{E}_{\mathrm{p}, \mathrm{U}}$ and $\boldsymbol{x}_{\mathrm{p}, \mathrm{L}}, \boldsymbol{x}_{\mathrm{p}, \mathrm{U}}$ represent the lower and upper bounds of $\boldsymbol{E}_{\mathrm{p}}$ and $\boldsymbol{x}_{\mathrm{p}}$, respectively. To illustrate effectiveness of the proposed method, the range of uncertain interval parameters are given as approximately $10 \%$ from the nominal value in the similar manner as the examples by Guest and Igusa (2008). Furthermore, since our purpose is to minimize the structural response at different robustness levels, we hereafter select the maximum and median structural responses as the desired percentiles to be minimized (Jekel and Haftka 2020), and the upper quartile, which is defined as the middle between the maximum and median, is also included to investigate the variation trend of objective function values and their statistical values. A small positive lower bound is given for a non-existing member to prevent numerical difficulty. The values of parameters in the following numerical examples are listed in Table 3, if not specified explicitly, where $I$ is a vector with all entries equal to 1 :

Table 3 Parameter setting of each example

\begin{tabular}{|c|c|c|c|}
\hline Parameters & Example 1 & Example 2 & Example 3 \\
\hline Upper bound volume $V_{\mathrm{U}}$ & $1 \mathrm{~m}^{3}$ & $0.2 \mathrm{~m}^{3}$ & $0.2 \mathrm{~m}^{3}$ \\
\hline Nominal value of each entry in $\overline{\boldsymbol{x}}_{\mathrm{p}}$ & $\begin{array}{c}\text { Values in Fig. } \\
5(\mathrm{~b})\end{array}$ & $\begin{array}{c}\text { Values in Fig. } \\
12(\mathrm{~b})\end{array}$ & Values in Fig. 18 \\
\hline Upper bound of $\boldsymbol{x}_{\mathrm{p}}$ & $\overline{\boldsymbol{x}}_{\mathrm{p}}+0.6 \boldsymbol{I}$ & $\overline{\boldsymbol{x}}_{\mathrm{p}}+0.1 \boldsymbol{I}$ & $\overline{\boldsymbol{x}}_{\mathrm{p}}+0.1 \boldsymbol{I}$ \\
\hline Lower bound of $\boldsymbol{x}_{\mathrm{p}}$ & $\overline{\boldsymbol{x}}_{\mathrm{p}}-0.6 \boldsymbol{I}$ & $\overline{\boldsymbol{x}}_{\mathrm{p}}-0.1 \boldsymbol{I}$ & $\overline{\boldsymbol{x}}_{\mathrm{p}}-0.1 \boldsymbol{I}$ \\
\hline Upper bound cross-sectional area $A_{\mathrm{U}}$ & $0.1 \mathrm{~m}^{2}$ & $0.05 \mathrm{~m}^{2}$ & $0.05 \mathrm{~m}^{2}$ \\
\hline Lower bound cross-sectional area $A_{\mathrm{L}}$ & \multicolumn{3}{|c|}{$1.0 \times 10^{-7} \mathrm{~m}^{2}$} \\
\hline Nominal value of each entry in $\overline{\boldsymbol{E}}_{\mathrm{p}}$ & $2.1 \times 10^{11} \mathrm{~Pa}$ \\
\hline Upper bound of $\boldsymbol{E}_{\mathrm{p}}$ & $1.1 \overline{\boldsymbol{E}}_{\mathrm{p}}$ \\
\hline Lower bound of $\boldsymbol{E}_{\mathrm{p}}$ & $0.9 \overline{\boldsymbol{E}}_{\mathrm{p}}$ \\
\hline Values of order $k$ in problem $(10)$ & $1,50,100$ \\
\hline Values of order $k$ in problem $(11)$ & $2,50,100$ \\
\hline Confidence level $\alpha_{k}$ & 0.9 \\
\hline Sample size $m$ & 200 \\
\hline
\end{tabular}

\subsection{Example 1}

The first example is a 14-node bridge frame with $6 \times 1$ grid and 31 beam elements, which is pinsupported at nodes 1 and 13. The initial ground structure is shown in Fig. 3. There are five downward loads with magnitude of $P=3 \times 10^{6} \mathrm{~N}$ applied at nodes $3,5,7,9$ and 11 . 


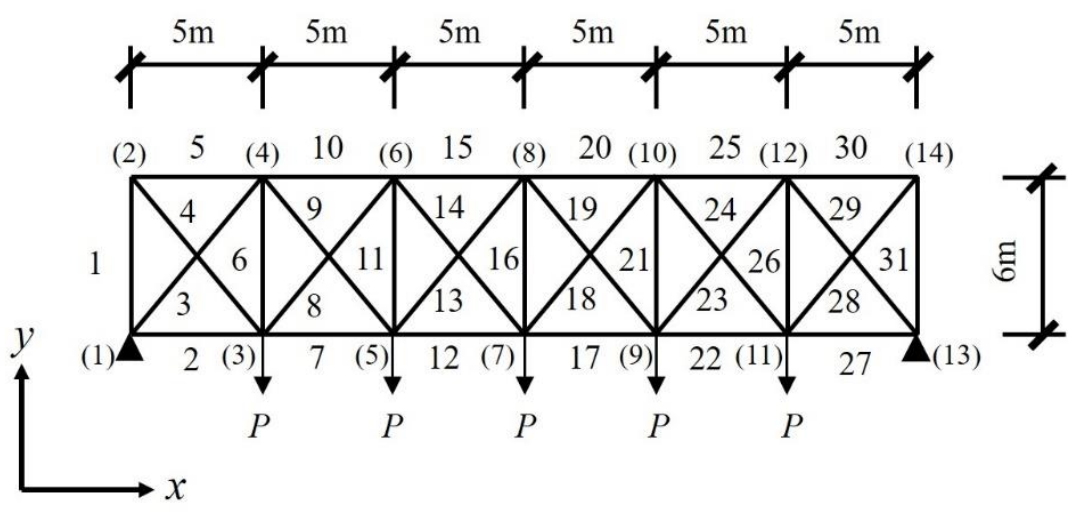

Fig. 3 Initial ground structure of Example 1

For verification of analysis and optimization programs, and investigating the differences between optimal design of truss and frame, we first minimize the structural volume under stress constraints on each element and $y$-directional displacement at each node except nodes 1 and 13. This problem has been studied by Rajan (1995) using truss elements. Note that uncertainty is not considered here, and Young's modulus for each beam element is $2.1 \times 10^{11} \mathrm{~Pa}$.

The design variables are the cross-sectional area of each element and the $y$-coordinates of the upper nodes 2, 4, 6, 8, 10, 12 and 14. Because the structural shape, loading conditions and boundary conditions are symmetric with respect to a $y$-directional line, the number of design variables is reduced from 38 to 20 , with 16 design variables $\boldsymbol{A}=\left(A_{1}, A_{2}, \ldots, A_{16}\right)$ representing the crosssectional areas of elements 1 to 16 , and four design variables $\boldsymbol{x}=\left(y_{2}, y_{4}, y_{6}, y_{8}\right)$ representing the $y$-coordinates of nodes $2,4,6$ and 8 . Since both bending moment and axial force exist in the frame structure, we have to ensure that both of the tensile and compressive stresses at the edge of element ends should not exceed the allowable stress. Therefore, we increase the upper bound of crosssectional area from $0.1 \mathrm{~m}^{2}$, which is used in Rajan (1995), to $0.2 \mathrm{~m}^{2}$ so that the cross-sectional area does not reach the upper bound.. The optimization problem to minimize the total structural volume $V(\boldsymbol{A}, \boldsymbol{x})$ is formulated as follows:

$$
\begin{array}{ll}
\text { Minimize } & V(\boldsymbol{A}, \boldsymbol{x}) \\
\text { subject to } & \delta_{i}(\boldsymbol{A}, \boldsymbol{x}) \leq \delta_{\mathrm{U}}, \quad i=2,3 \ldots, 12,14 \\
& \left\{\begin{array}{l}
\sigma_{l}^{\max -\mathrm{t}}(\boldsymbol{A}, \boldsymbol{x}) \leq \sigma_{\mathrm{t}, \mathrm{U}} \\
\sigma_{l}^{\max -\mathrm{c}}(\boldsymbol{A}, \boldsymbol{x}) \leq \sigma_{\mathrm{c}, \mathrm{U}}
\end{array}\right. \\
& y_{\mathrm{L}} \leq y_{\mathrm{L}} \leq y_{\mathrm{U}}, \quad j=2,4,6,8
\end{array}
$$

where $\delta_{i}(\boldsymbol{A}, \boldsymbol{x})$ is the downward displacement of node $i$, and $\delta_{\mathrm{U}}=0.1 \mathrm{~m}$ is its upper bound; $\sigma_{l}^{\text {max-t }}(\boldsymbol{A}, \boldsymbol{x})$ and $\sigma_{l}^{\text {max-c }}(\boldsymbol{A}, \boldsymbol{x})$ represent the maximum tension and compressive edge stresses $(>0)$ of $l$ th element, and $\sigma_{\mathrm{t}, \mathrm{U}}=1.30 \times 10^{8} \mathrm{~Pa}$ and $\sigma_{\mathrm{c}, \mathrm{U}}=1.04 \times 10^{8} \mathrm{~Pa}$ are the upper bounds of 
edge stress in tension and compression, respectively. Bounds for the variables are $y_{\mathrm{L}}=2 \mathrm{~m}$, $y_{\mathrm{U}}=8 \mathrm{~m}, A_{\mathrm{U}}=0.2 \mathrm{~m}^{2}$, and $A_{\mathrm{L}}=1.0 \times 10^{-7} \mathrm{~m}^{2}$.

Problem (12) is solved by GA, and the optimal solution is shown in Fig. 5(a). The structural volume is $5.42 \mathrm{~m}^{3}$, which is about $6.4 \%$ larger than the result in Rajan (1995). The main reason is that the truss structure has only axial force and the element is either under uniaxial tension or compression. On the other hand, the edge stress of frame element consists of bending stress and uniaxial tensile or compressive stress, which is illustrated in Fig. 4, and the cross-sectional area need to be larger than that of the truss element in order not to violate the stress constraints, leading to a little increase of the total structural volume.

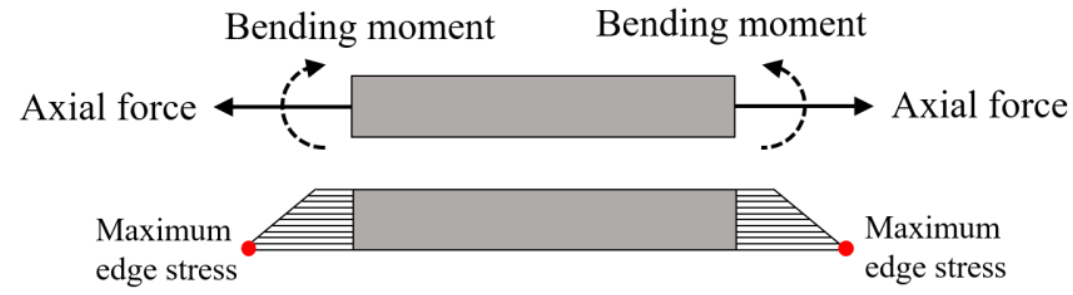

Fig. 4 Illustration of internal force and maximum edge stress of beam element

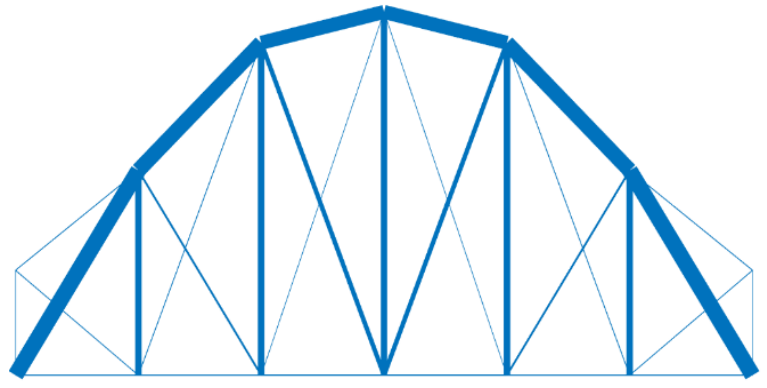

(a)

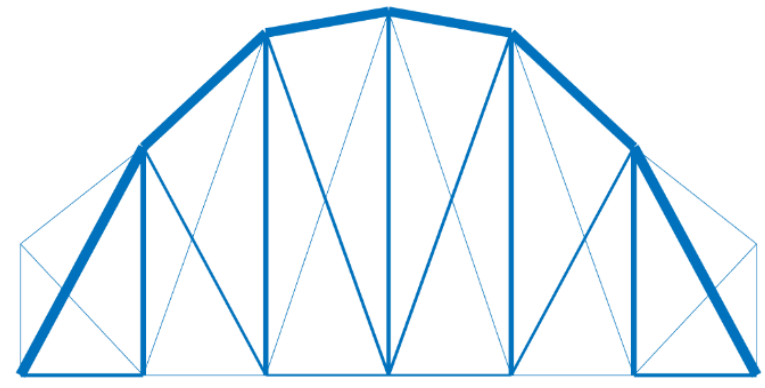

(b)

Fig. 5 Optimal solutions of Example 1: (a) problem (12); (b) problem (13)

Next, we select Young's modulus of each element and $x$ - and $y$-coordinates of nodes 2, 4, 6, 8, 10, 12 and 14 as uncertain parameters, and minimize the maximum stress under volume constraint. The initial structure is the same as shown in Fig. 3 and design variables are $\boldsymbol{A}=\left(A_{1}, A_{2}, \ldots, A_{16}\right)$ and $\boldsymbol{x}=\left(y_{2}, y_{4}, y_{6}, y_{8}\right)$. Using the parameter values listed in Table 3 , the optimization problem is formulated as follows: 
Minimize $\sigma^{\max }=\max _{i=1,2, \ldots, 16} \sigma_{i}^{\max }\left(\boldsymbol{A}, \boldsymbol{x} ; \overline{\boldsymbol{E}}_{\mathrm{p}}\right)$

subject to $V(\boldsymbol{A}, \boldsymbol{x}) \leq V_{\mathrm{U}}$

$$
A_{\mathrm{L}} \leq A_{l} \leq A_{\mathrm{U}}, \quad l=1,2, \ldots, 16 ; y_{\mathrm{L}} \leq y_{j} \leq y_{\mathrm{U}}, j=2,4,6,8
$$

Problem (13) is solved using GA and the optimal solution is shown in Fig. 5(b). Then, based on the optimal shape in Fig. 5(b) we choose the cross-sectional areas as design variables and consider the variation in uncertain parameters, and the multiobjective optimization problem reads

Minimize $\sigma^{\max }\left(\boldsymbol{A} ; \overline{\boldsymbol{E}}_{\mathrm{p}}, \overline{\boldsymbol{x}}_{\mathrm{p}}\right), \quad \sigma_{1: 200}^{\max }\left(\boldsymbol{A} ; \boldsymbol{E}_{\mathrm{p}}, \boldsymbol{x}_{\mathrm{p}}\right), \quad \sigma_{50: 200}^{\max }\left(\boldsymbol{A} ; \boldsymbol{E}_{\mathrm{p}}, \boldsymbol{x}_{\mathrm{p}}\right), \quad \sigma_{100: 200}^{\max }\left(\boldsymbol{A} ; \boldsymbol{E}_{\mathrm{p}}, \boldsymbol{x}_{\mathrm{p}}\right)$

subject to $V(\boldsymbol{A}) \leq V_{\mathrm{U}} ; A_{\mathrm{L}} \leq A_{l} \leq A_{\mathrm{U}}, \quad l=1,2, \ldots, 16$

As a result of optimization, the 200 solutions converged to a set of 70 different Pareto optimal solutions, and the total computational efforts for solving problems (13) and (14), including the iteration steps, number of function analyses and computation time, are listed in Table 4. Since finite element analysis can be avoided if the individuals in the current population have appeared in the previous iteration and thus computational efforts can be saved, the following criteria is adopted to determine whether the analysis for the current individual is needed or not:

$$
\frac{\left\|\boldsymbol{p}_{1}-\boldsymbol{p}_{i}\right\|}{\left\|\boldsymbol{p}_{i}\right\|} \leq 0.001, i=1,2, \ldots, n_{\mathrm{a}}
$$

where $\boldsymbol{p}_{1}$ is the current individual to be evaluated, $\boldsymbol{p}_{i}$ is the individual that has appeared before and $n_{\mathrm{a}}$ is number of individuals that have appeared before without duplication. By using Eq. (15) the number of analyses for solving problems (13) and (14) without duplication can be obtained as listed in Table 4 . Note that the computation time is calculated for the total number of analyses.

Table 4 Computational efforts of Examples 1, 2 and 3

\begin{tabular}{|c|c|c|c|c|}
\hline Example & $\begin{array}{c}\text { Iteration } \\
\text { steps }\end{array}$ & Number of analyses & $\begin{array}{c}\text { Number of analyses } \\
\text { without duplication }\end{array}$ & $\begin{array}{c}\text { Computation } \\
\text { time }\end{array}$ \\
\hline 1 & 621 & $\begin{array}{c}400,200(\text { problem 13) } \\
7,880,000(\text { problem 14) }\end{array}$ & $\begin{array}{c}45,533 \text { (problem 13) } \\
4,345,000(\text { problem 14) }\end{array}$ & $4243.78 \mathrm{sec}$ \\
\hline 2 & 951 & $\begin{array}{c}115,600(\text { problem 17) } \\
15,320,000(\text { problem 18) }\end{array}$ & $\begin{array}{c}37,605(\text { problem 17) } \\
11,302,600(\text { problem 18) }\end{array}$ & $5782.27 \mathrm{sec}$ \\
\hline 3 & 593 & $\begin{array}{c}88,200(\text { problem 22) } \\
6,120,000(\text { problem 23) }\end{array}$ & $\begin{array}{c}40,420(\text { problem 22) } \\
4,311,400(\text { problem 23) }\end{array}$ & $3156.42 \mathrm{sec}$ \\
\hline
\end{tabular}

The Pareto optimal solutions at different steps are plotted in Figs. 6, 7 and 8, respectively, in the planes of $\sigma^{\max }$ and one of $\sigma_{1: 200}^{\max }, \sigma_{50: 200}^{\max }$ and $\sigma_{100: 200}^{\max }$, where the solutions on the Pareto front are denoted by PF followed by the step number in the parentheses. It can be observed from Fig. 6(a) that the Pareto front is generated with good accuracy after step 30, and the optimal value of $\sigma^{\max }$ remains the same due to the addition of optimal solution of problem (13) into the initial population of problem (14). Fig. 6(b) plots the detailed view of the values of Pareto optimal solutions and 
their corresponding trade-off relationships. We can see that variation of $\sigma_{1: 200}^{\max }$ at the Pareto front is greater than that of $\sigma^{\max }$. The same conclusions can be drawn from Figs. 7 and 8.

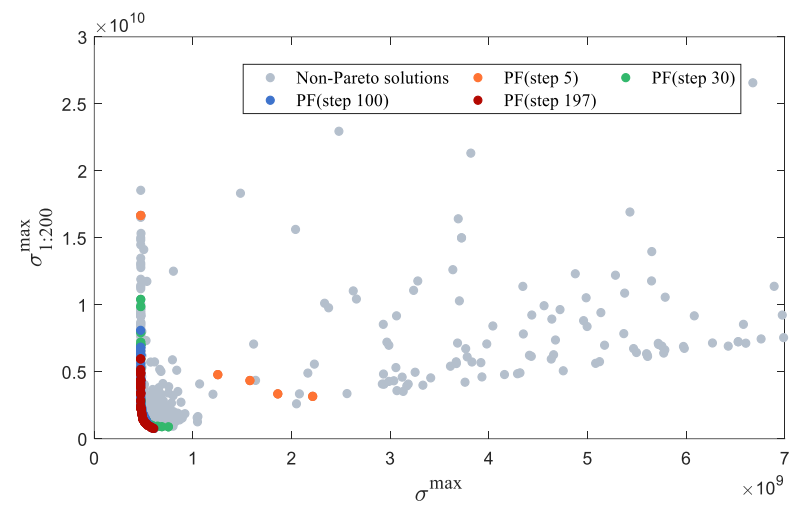

(a)

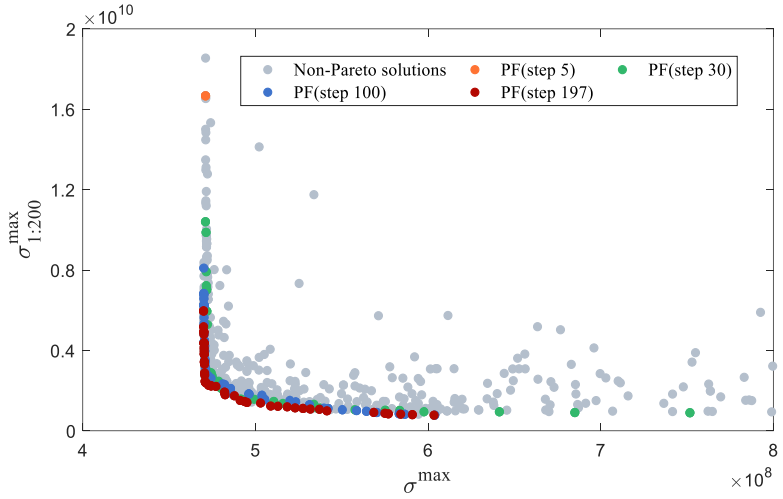

(b)

Fig. 6 Stepwise Pareto front of objectives $\sigma^{\max }$ and $\sigma_{1: 200}^{\max }$ : (a) Overall review; (b) Detailed view

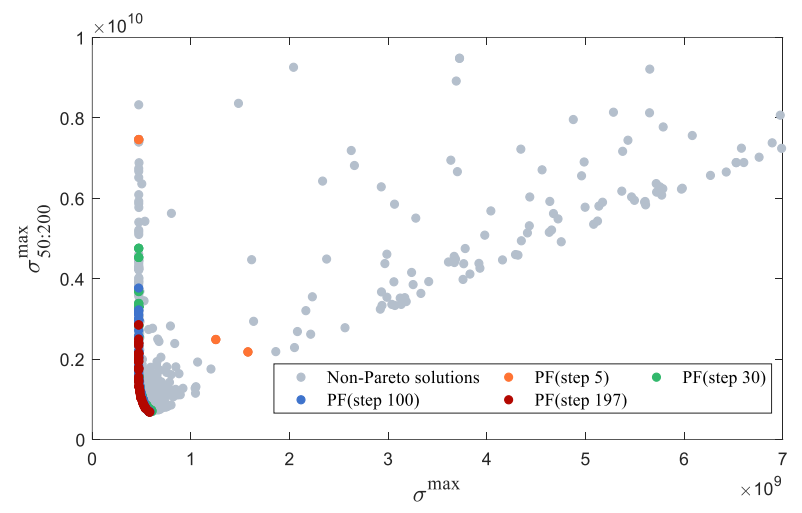

(a)

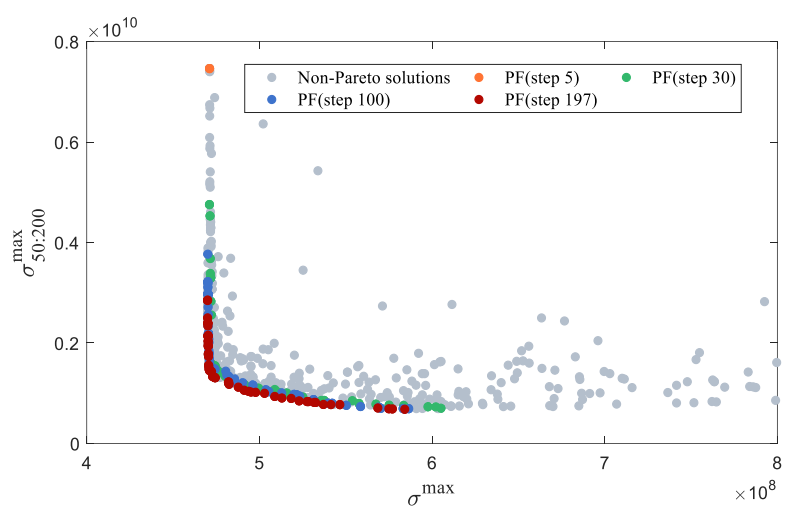

(b)

Fig. 7 Stepwise Pareto front of objectives $\sigma^{\max }$ and $\sigma_{50: 200}^{\max }$ : (a) Overall review; (b) Detailed view

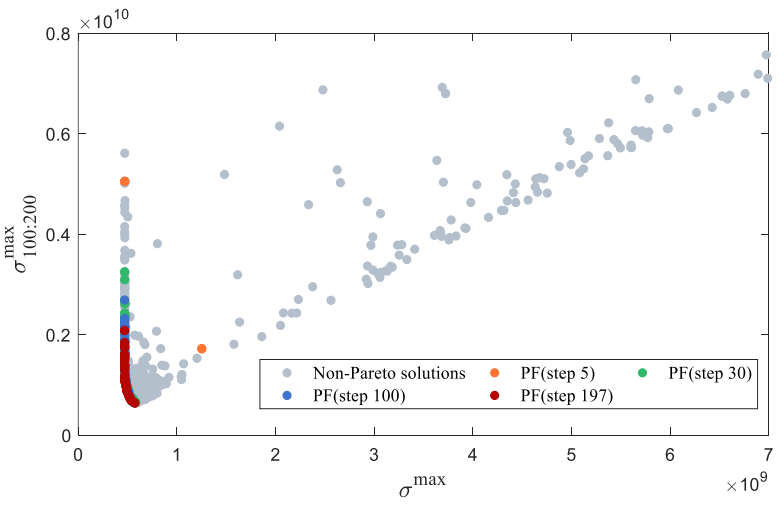

(a)

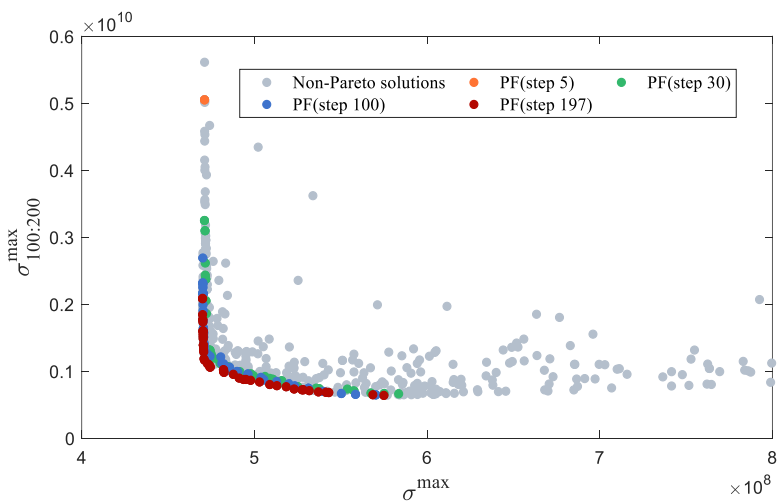

(b)

Fig. 8 Stepwise Pareto front of objectives $\sigma^{\max }$ and $\sigma_{100: 200}^{\max }$ : (a) Overall review; (b) Detailed view 
The Pareto optimal solutions that have the smallest values of $\sigma^{\max }, \sigma_{1: 200}^{\max }, \sigma_{50: 200}^{\max }$ and $\sigma_{100: 200}^{\max }$, respectively, are denoted by solutions A, B, C and D, and shown in Fig. 9. The maximum edge stresses of each element along its length, which is calculated without considering uncertainty, are also depicted. Note that the thin elements whose cross-sectional areas less than $0.0001 \mathrm{~m}^{2}$ are removed from the optimal solutions in Fig. 9, and their objective values before and after removing the thin elements are listed in Tables 5 and 6, respectively, together with their structural volume. The distribution of cross-sectional areas are plotted in Fig. 10.

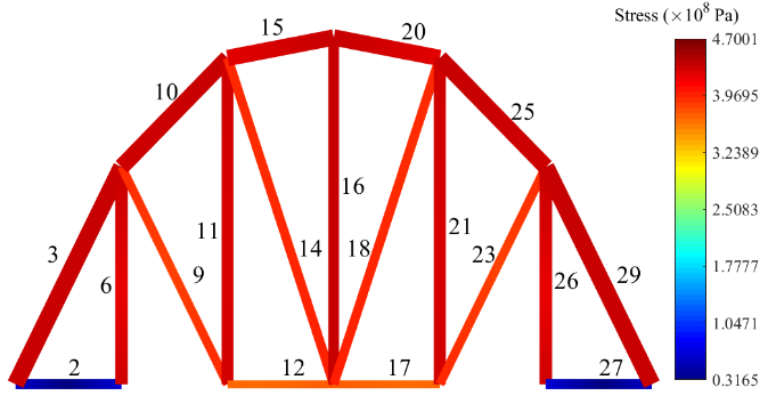

(a)

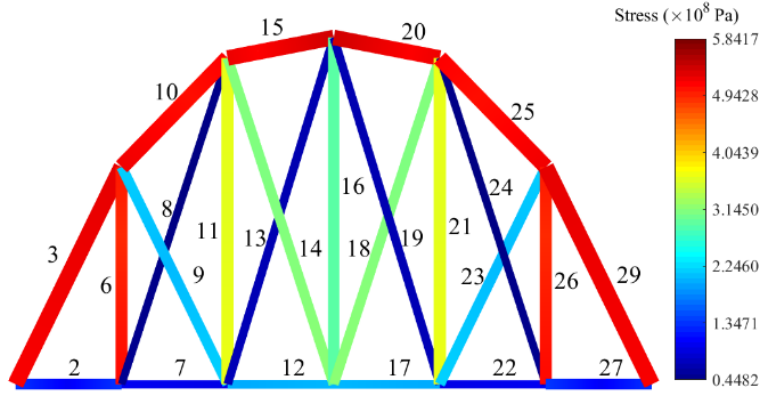

(c)

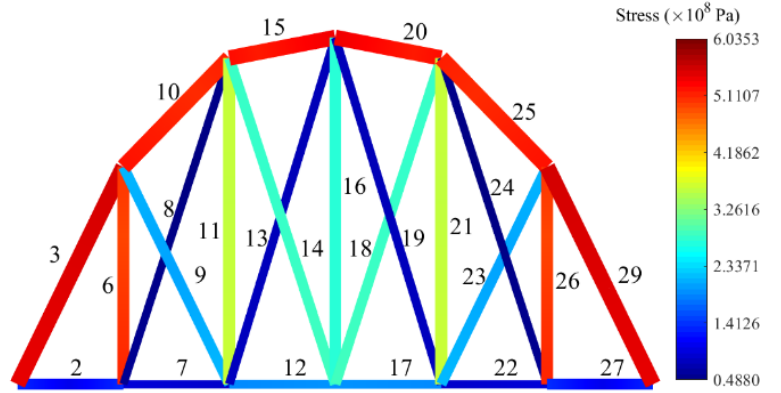

(b)

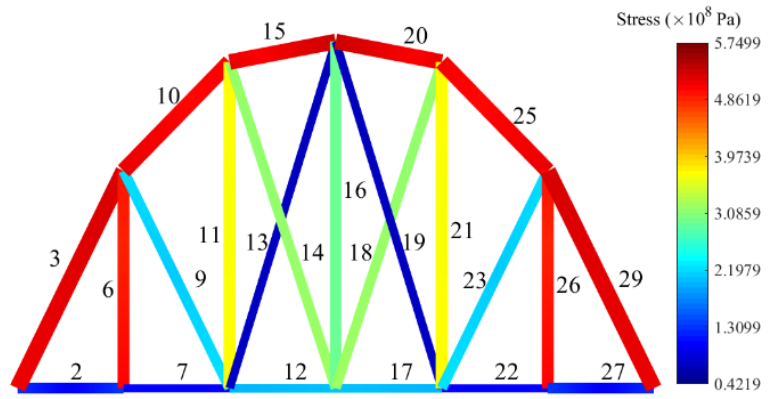

(d)

Fig. 9 Pareto optimal solutions of problem (14): (a) Solution A; (b) Solution B; (c) Solution C; (d) Solution D 


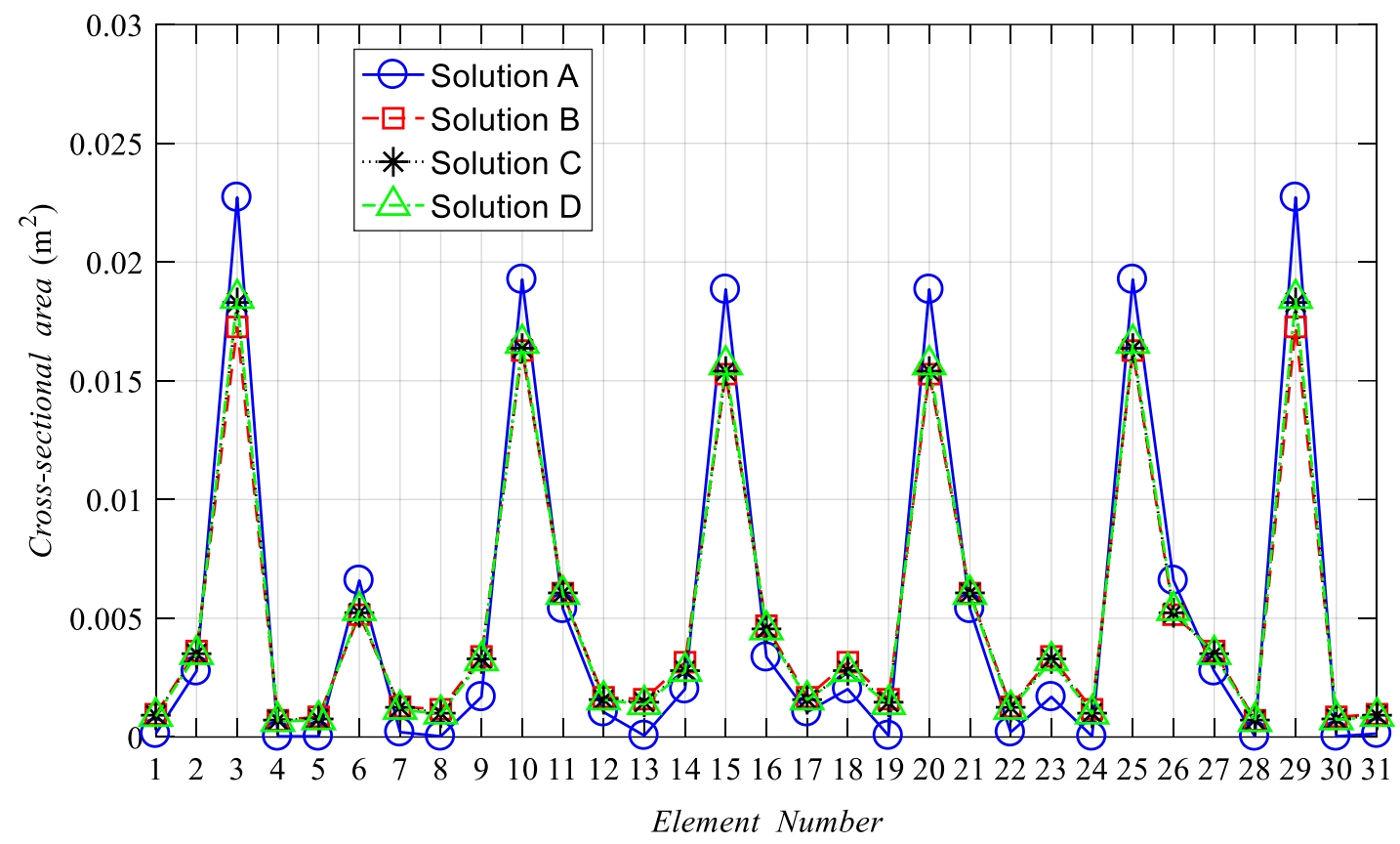

Fig. 10 Distribution of cross-sectional areas of solutions A, B, C and D of problem (14) in Example 1

Table 5. Objective values and structural volume of solutions A, B, C and D of problem (14) in Example 1 before removing thin elements

\begin{tabular}{|c|c|c|c|c|c|}
\hline Solution & $\sigma^{\max }(\mathrm{Pa})$ & $\sigma_{1: 200}^{\max }(\mathrm{Pa})$ & $\sigma_{50: 200}^{\max }(\mathrm{Pa})$ & $\sigma_{100: 200}^{\max }(\mathrm{Pa})$ & Volume $\left(\mathrm{m}^{3}\right)$ \\
\hline $\mathrm{A}$ & $4.7001 \times 10^{8}$ & $5.9733 \times 10^{9}$ & $2.8549 \times 10^{9}$ & $2.0861 \times 10^{9}$ & 1.0 \\
\hline $\mathrm{B}$ & $6.0353 \times 10^{8}$ & $7.7531 \times 10^{8}$ & $6.9560 \times 10^{8}$ & $6.5546 \times 10^{8}$ & 1.0 \\
\hline $\mathrm{C}$ & $5.8417 \times 10^{8}$ & $8.2934 \times 10^{8}$ & $6.8127 \times 10^{8}$ & $6.4418 \times 10^{8}$ & 1.0 \\
\hline $\mathrm{D}$ & $5.7499 \times 10^{8}$ & $8.7466 \times 10^{8}$ & $6.9079 \times 10^{8}$ & $6.3814 \times 10^{8}$ & 1.0 \\
\hline
\end{tabular}

Table 6. Objective values and structural volume of solutions A, B, C and D of problem (14) in Example 1 after removing thin elements

\begin{tabular}{|c|c|c|c|c|c|}
\hline Solution & $\sigma^{\max }(\mathrm{Pa})$ & $\sigma_{1: 200}^{\max }(\mathrm{Pa})$ & $\sigma_{50: 200}^{\max }(\mathrm{Pa})$ & $\sigma_{100: 200}^{\max }(\mathrm{Pa})$ & Volume $\left(\mathrm{m}^{3}\right)$ \\
\hline $\mathrm{A}$ & $4.7080 \times 10^{8}$ & $1.7213 \times 10^{10}$ & $7.3785 \times 10^{9}$ & $4.1258 \times 10^{9}$ & 1.0 \\
\hline $\mathrm{B}$ & $6.0929 \times 10^{8}$ & $7.7872 \times 10^{8}$ & $6.9805 \times 10^{8}$ & $6.6848 \times 10^{8}$ & 1.0 \\
\hline $\mathrm{C}$ & $5.8482 \times 10^{8}$ & $8.3908 \times 10^{8}$ & $6.8469 \times 10^{8}$ & $6.4775 \times 10^{8}$ & 1.0 \\
\hline $\mathrm{D}$ & $5.8070 \times 10^{8}$ & $8.8895 \times 10^{8}$ & $6.9200 \times 10^{8}$ & $6.4595 \times 10^{8}$ & 1.0 \\
\hline
\end{tabular}

We can see from Fig. 9 and Tables 5 and 6 that solution A has obviously large values of objectives $\sigma_{1: 200}^{\max }, \sigma_{50: 200}^{\max }$ and $\sigma_{100: 200}^{\max }$. Solution B has the minimum value of $\sigma_{1: 200}^{\max }$ and some of the elements, which do not exist in solution A, have moderately large cross-sectional areas to reduce 
the effect of uncertainties in Young's modulus and nodal locations, resulting in the large stiffness of the frame.

As seen from Tables 5 and 6, the objective values of solutions B, C, and D as well as the objective value of $\sigma^{\max }$ in solution A before and after removing thin elements are very close. However, the objective values of $\sigma_{1: 200}^{\max }, \sigma_{50: 200}^{\max }$ and $\sigma_{100: 200}^{\max }$ in solution A after removing thin elements are greater than those before removal. This is mainly because although solution A has the minimum value of $\sigma^{\max }$ among 70 Pareto optimal solutions, it is unstable if bending stiffness is very small and become worse when the thin elements are removed, making it more sensitive to the uncertainties, and thus its maximum stress increase rapidly as deformation becomes asymmetric.

It is confirmed that solutions $\mathrm{C}$ and $\mathrm{D}$ have the smallest values of $\sigma_{50: 200}^{\max }$ and $\sigma_{100: 200}^{\max }$, respectively, and elements 8 and 24 are removed in solution D. We can also observe from Tables 5 and 6 that the nominal objective value $\sigma^{\max }$ decrease as $k$ is increased. However, the solutions $\mathrm{A}$ and $\mathrm{D}$ that minimize the nominal and median values are very different, indicating that a small asymmetric property leads to a large increase of the maximum stress, and accordingly leads to a significant difference in the optimal solutions. By contrast, the extreme value $\sigma_{1: 200}^{\max }$ increases as $k$ is increased. However, the increase is not very significant. The cross-sectional areas of solutions $\mathrm{B}, \mathrm{C}$ and $\mathrm{D}$ have similar distribution as shown in Fig. 10.

Solutions A, B, C and D have the same structural volume of $1.0 \mathrm{~m}^{3}$, however; they have different distribution of cross-sectional areas after solving problem (14), leading to different performances on minimizing the maximum stress. Furthermore, it can be seen that none of these four solutions has minimum values for any of the two objectives, indicating that the optimal solution may vary with robustness level, and the designers can appropriately choose the optimal solution according to the various robustness levels.

Moreover, in order to further investigate the effect of relaxing worst-case value of the maximum value on the optimal solutions, the worst-case value is approximated by order statistics with a larger confidence level $\alpha_{k}=0.99$ and $\beta=0.99$, and the minimum sample size that the worst sample (i.e., $k=1$ ) satisfies $\alpha_{k}=0.99$ and $\beta=0.99$ is 459 (Yamakawa and Ohsaki 2016). The following optimization problem is formulated and solved by GA with the same parameter values as in problem (13):

$$
\begin{array}{ll}
\text { Minimize } & \sigma_{1: 459}^{\max }\left(\boldsymbol{A} ; \boldsymbol{E}_{\mathrm{p}}, \boldsymbol{x}_{\mathrm{p}}\right) \\
\text { subject to } & V(\boldsymbol{A}) \leq V_{\mathrm{U}} ; A_{\mathrm{L}} \leq A_{l} \leq A_{\mathrm{U}}, \quad l=1,2, \ldots, 16
\end{array}
$$

The optimal solution of problem (16) is shown in Fig. 11, where the maximum stresses for each element are calculated without considering uncertainty. The optimal value of $\sigma_{1: 459}^{\max }$ is 7.9923 $\times 10^{8} \mathrm{~Pa}$, which is a little larger than the objective value of $\sigma_{1: 200}^{\max }$ of Solution B due to the trade-off relationship between robustness level and minimizing the maximum stress. Note that the value of $\beta$ for $\sigma_{1: 200}^{\max }$ at confidence level $\alpha_{k}=0.99$ is 0.977. Compared to solution B in Fig. 9(b), it can be seen 
that the cross-sectional areas of elements 1, 4, 5, 28, 30 and 31, which do not exist in solution B, have moderately large values in Fig. 11 to reduce the extreme worst-case value, resulting in different performance on minimizing the maximum stress in the structure. Since the extreme worst-case is a rare event and it may cost more to build a structure which satisfies the requirement of large worst-case value of maximum stress, in the present study we relax the worst-case value to some extent and assign the sample size $m=200$, and the computational effort will also be saved.

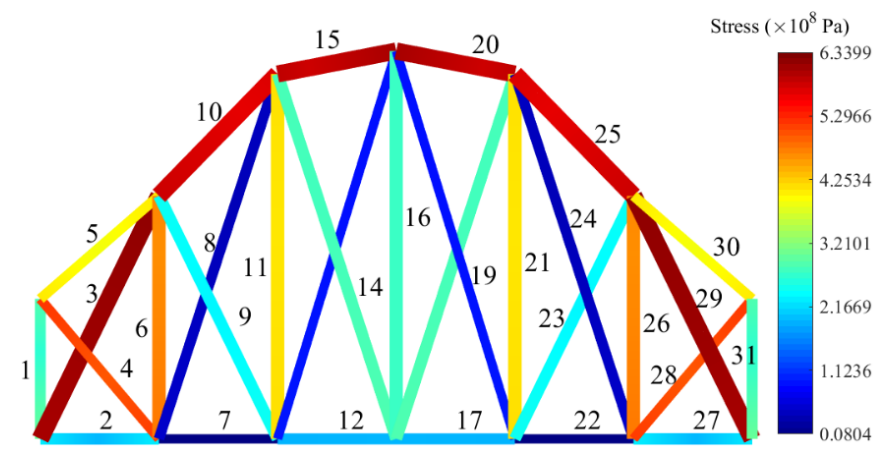

Fig. 11 Optimal solution of problem (16)

\subsection{Example 2}

Example 2 involves a $4 \times 1$ grid with 10 nodes and 21 elements, and a downward vertical load $P$ with magnitude of $100 \mathrm{~N}$ is applied at node 5. The structure is pin-supported at nodes 1 and 9 , and the geometry of the structure is shown in Fig. 12(a). The design variables are the cross-sectional areas of elements 1 to $11 \boldsymbol{A}=\left(A_{1}, A_{2}, \ldots A_{11}\right)$ incorporating symmetry, and the uncertain parameters are Young's modulus of each element and $x$ - and $y$-coordinates of nodes 2, 3, 4, 6, 7, 8 and 10.
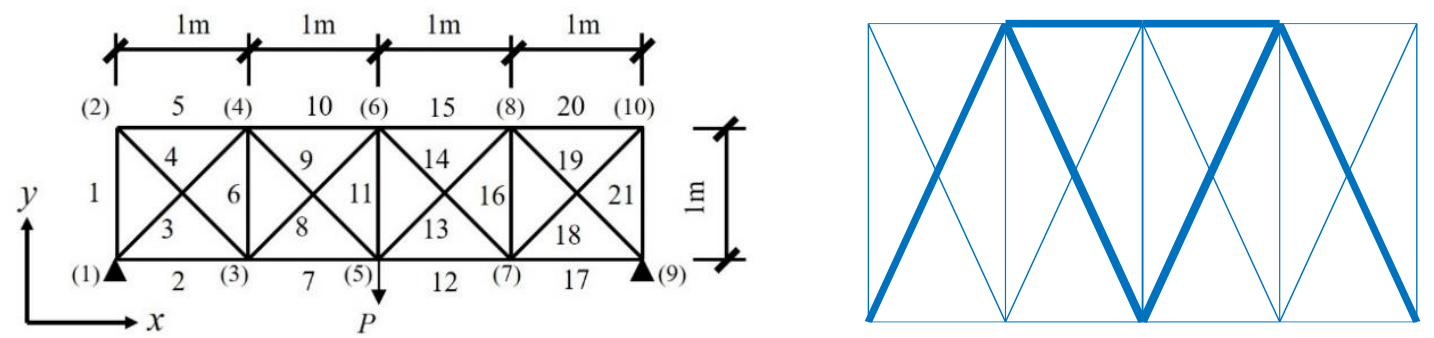

Fig. 12 (a) Initial ground structure of Example 2; (b) Optimal solution of problem (17)

First, in accordance with Eq. (9), the following single objective optimization problem to minimize the maximum stress is solved 


$$
\begin{aligned}
& \text { Minimize } \sigma^{\max }=\max _{i=1,2, \ldots, 11} \sigma_{i}^{\max }\left(\boldsymbol{A}, \overline{\boldsymbol{E}}_{\mathrm{p}}\right) \\
& \text { subject to } V(\boldsymbol{A}) \leq V_{\mathrm{U}} ; A_{\mathrm{L}} \leq A_{l} \leq A_{\mathrm{U}}, \quad l=1,2, \ldots, 11
\end{aligned}
$$

In a similar manner as Example 1, based on the optimal solution of problem (17) we formulate the following multiobjective optimization problem:

$$
\begin{array}{ll}
\text { Minimize } & \sigma^{\max }\left(\boldsymbol{A}, \overline{\boldsymbol{E}}_{\mathrm{p}}, \overline{\boldsymbol{x}}_{\mathrm{p}}\right), \sigma_{1: 200}^{\max }\left(\boldsymbol{A}, \boldsymbol{E}_{\mathrm{p}}, \boldsymbol{x}_{\mathrm{p}}\right), \sigma_{50: 200}^{\max }\left(\boldsymbol{A}, \boldsymbol{E}_{\mathrm{p}}, \boldsymbol{x}_{\mathrm{p}}\right), \sigma_{100: 200}^{\max }\left(\boldsymbol{A}, \boldsymbol{E}_{\mathrm{p}}, \boldsymbol{x}_{\mathrm{p}}\right) \\
\text { subject to } & V(\boldsymbol{A}) \leq V_{\mathrm{U}} ; A_{\mathrm{L}} \leq A_{l} \leq A_{\mathrm{U}}, \quad l=1,2, \cdots 11
\end{array}
$$

The optimal solution of problem (17) using the parameter values in Table 3 is shown in Fig. 12(b), and the four solutions A, B, C and D from the 70 Pareto optimal solutions of problem (18), which have the minimum objective values of $\sigma^{\max }, \sigma_{1: 200}^{\max }, \sigma_{50: 200}^{\max }$ and $\sigma_{100: 200}^{\max }$, respectively, are presented in Fig. 13, together with the corresponding maximum edge stress obtained without considering uncertainty. The computational efforts for solving problems (17) and (18) are listed in Table 4. The elements with cross-sectional areas less than $0.0001 \mathrm{~m}^{2}$ have been removed from Fig, 13, and the distribution of cross-sectional areas are plotted in Fig. 14. As seen from Figs. 13 and 14, although solutions B, C and D have the same structural topology, the distribution of cross-sectional areas are not exactly the same.

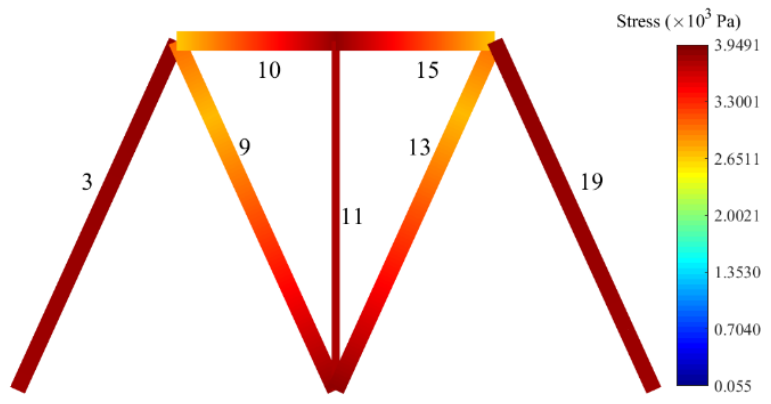

(a)

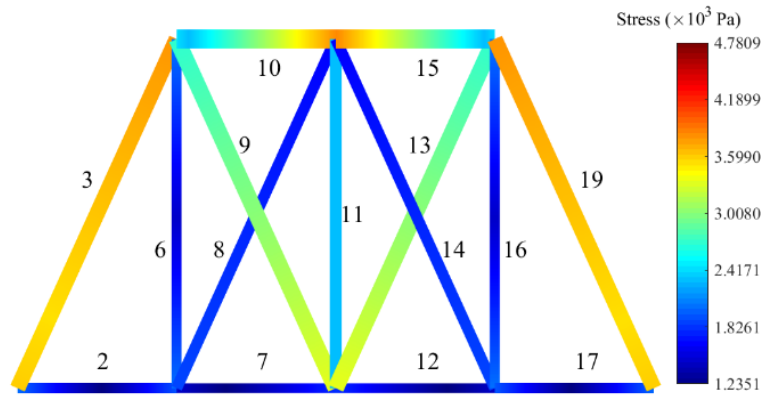

(c)

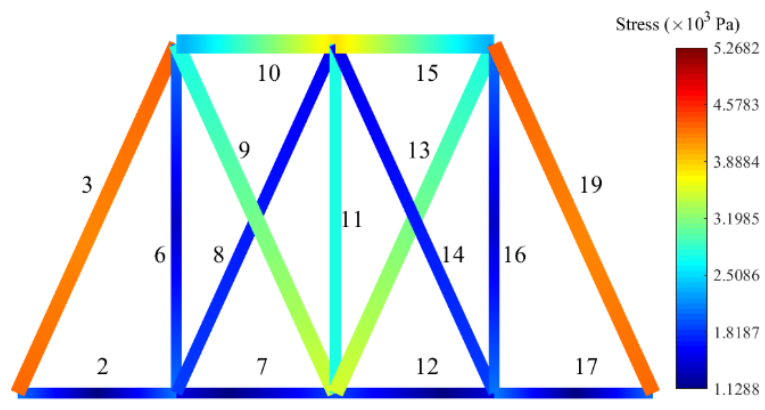

(b)

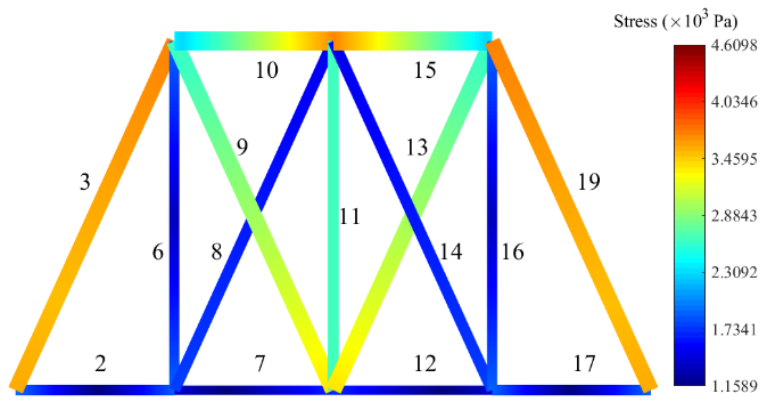

(d)

Fig. 13 Pareto optimal solutions of problem (18): (a) Solution A; (b) Solution B; (c) Solution C; (d) Solution D 


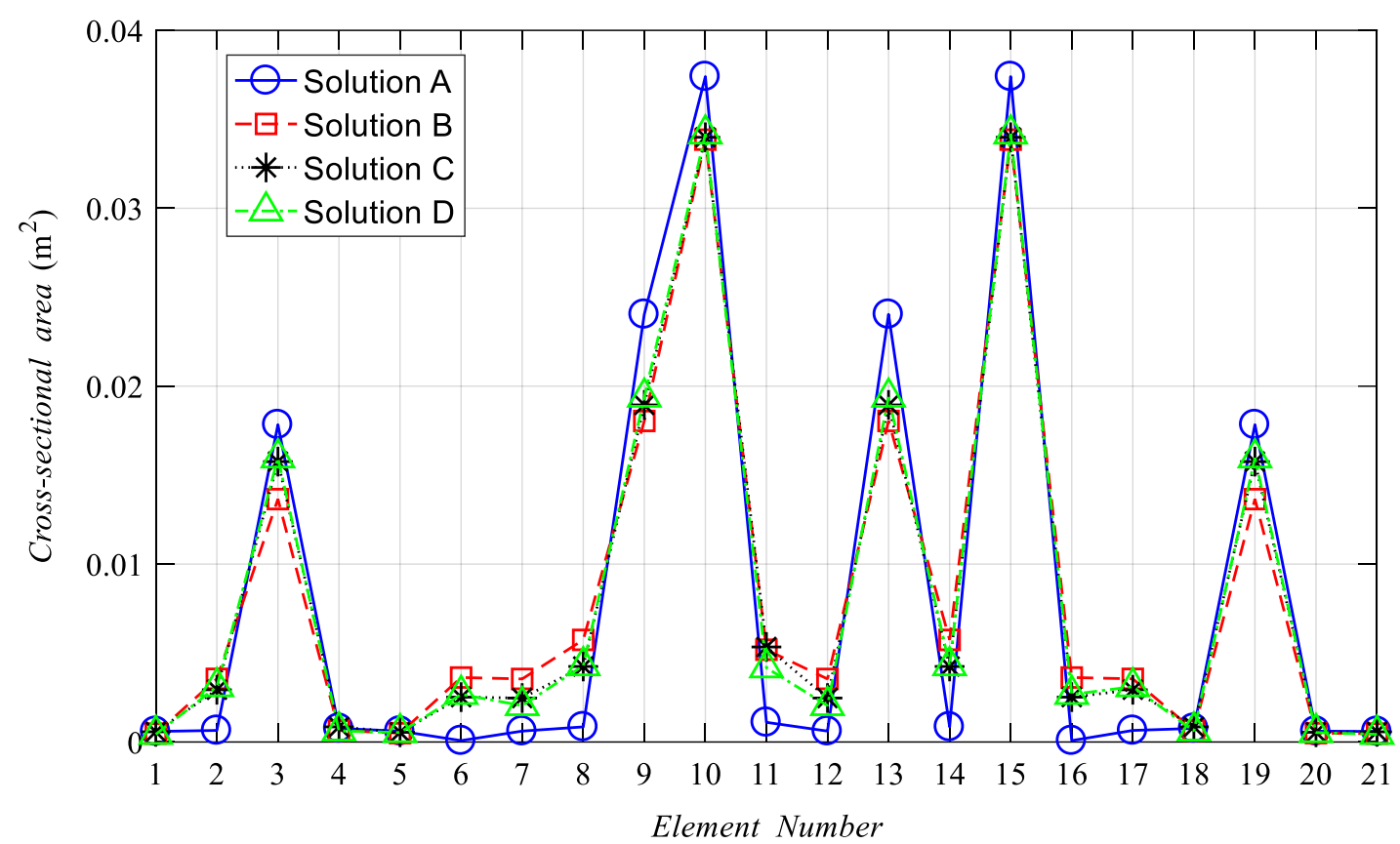

Fig. 14 Distribution of cross-sectional areas of solutions A, B, C and D of problem (18) in Example 2

Table 7. Objective value and structural volume of solutions A, B, C and D of problem (18) in Example 2 before removing thin elements

\begin{tabular}{|c|c|c|c|c|c|}
\hline Solution & $\sigma^{\max }(\mathrm{Pa})$ & $\sigma_{1: 200}^{\max }(\mathrm{Pa})$ & $\sigma_{50: 200}^{\max }(\mathrm{Pa})$ & $\sigma_{100: 200}^{\max }(\mathrm{Pa})$ & Volume $\left(\mathrm{m}^{3}\right)$ \\
\hline $\mathrm{A}$ & 3949.13 & 15475.38 & 9526.87 & 7193.64 & 0.2 \\
\hline $\mathrm{B}$ & 5268.16 & 6618.92 & 5984.07 & 5615.98 & 0.2 \\
\hline $\mathrm{C}$ & 4780.90 & 6998.41 & 5742.66 & 5268.61 & 0.2 \\
\hline $\mathrm{D}$ & 4609.75 & 7091.41 & 5842.31 & 5178.45 & 0.2 \\
\hline
\end{tabular}

Table 7 lists the objective values and structural volume of solutions A, B, C and D before removing thin elements. It can be observed from the table that for solution A the objective values of three order statistics are far greater than its nominal objective value. This is mainly because without considering variation in uncertain parameters the final optimal structure, as shown in Fig. 13(a), is unstable, if bending stiffness is neglected, and more sensitive to the uncertainty. On the other hand, with the re-distribution procedure of cross-sectional areas, solutions B, C and D have reduced the maximum stress under uncertainty at the cost of increasing the maximum stress for nominal uncertain parameters. Moreover, among these four solutions the decrease from $\sigma_{1: 200}^{\max }$ to $\sigma_{50: 200}^{\max }$ is larger than the decrease from $\sigma_{50: 200}^{\max }$ to $\sigma_{100: 200}^{\max }$, indicating that the decrease of order statistics with respect to $k$ for large order $k$ may be small. 
Based on Eq. (11), we further formulate the following optimization problem by using trimmed means as objective functions:

$$
\begin{aligned}
& \text { Minimize } \sigma^{\max }\left(\boldsymbol{A} ; \overline{\boldsymbol{E}}_{\mathrm{p}}, \overline{\boldsymbol{x}}_{\mathrm{p}}\right), \hat{\sigma}_{2: 200}^{\max }\left(\boldsymbol{A} ; \boldsymbol{E}_{\mathrm{p}}, \boldsymbol{x}_{\mathrm{p}}\right), \hat{\sigma}_{50: 200}^{\max }\left(\boldsymbol{A} ; \boldsymbol{E}_{\mathrm{p}}, \boldsymbol{x}_{\mathrm{p}}\right), \hat{\sigma}_{100: 200}^{\max }\left(\boldsymbol{A} ; \boldsymbol{E}_{\mathrm{p}}, \boldsymbol{x}_{\mathrm{p}}\right) \\
& \text { subject to } V(\boldsymbol{A}) \leq V_{\mathrm{U}} ; \quad A_{\mathrm{L}} \leq A_{l} \leq A_{\mathrm{U}}, \quad l=1,2, \ldots, 11
\end{aligned}
$$

where

$$
\hat{\sigma}_{k: 200}^{\max }\left(\boldsymbol{A} ; \boldsymbol{E}_{\mathrm{p}}, \boldsymbol{x}_{\mathrm{p}}\right)=\frac{1}{3}\left(\sigma_{k-1: 200}^{\max }+\sigma_{k: 200}^{\max }+\sigma_{k+1: 200}^{\max }\right), \quad k=2,50,100
$$

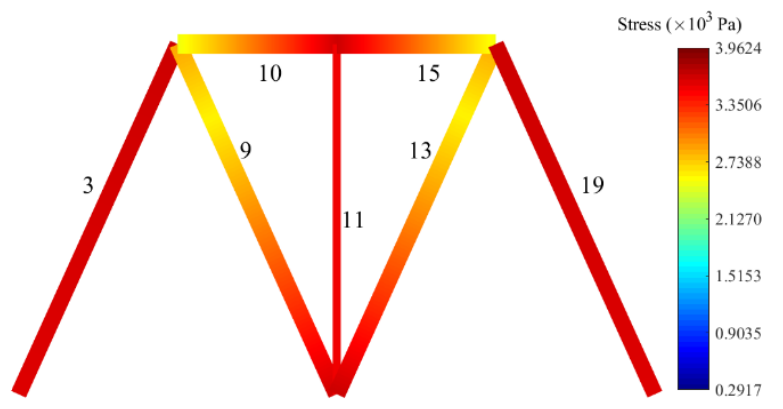

(a)

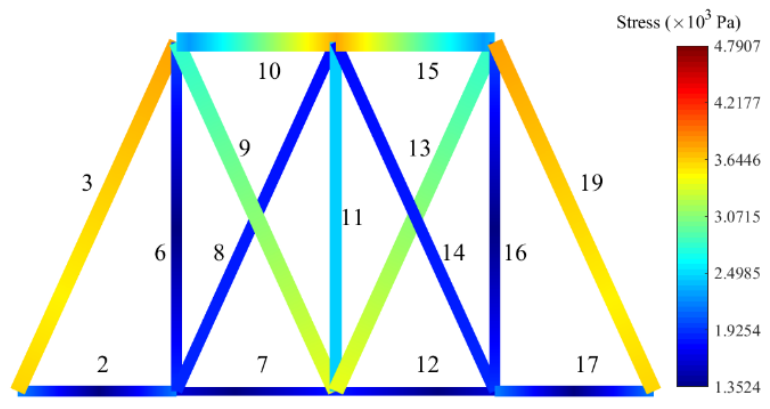

(c)

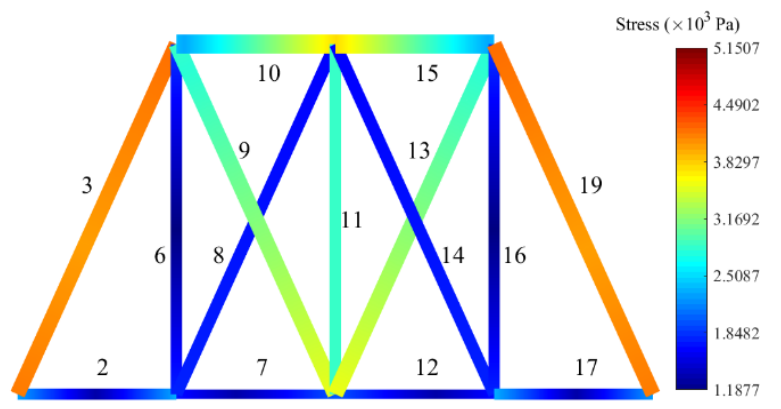

(b)

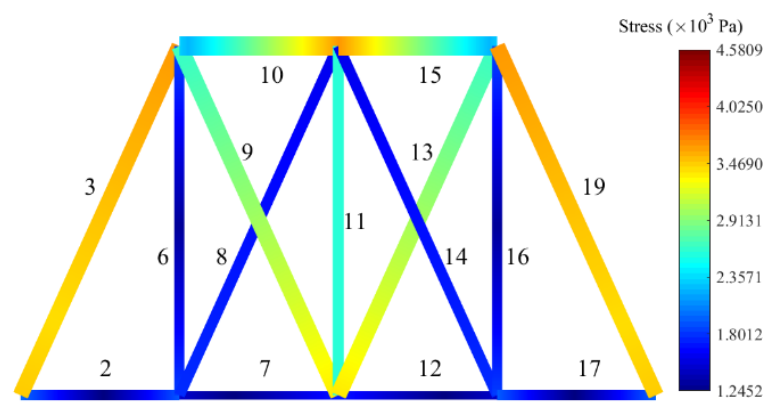

(d)

Fig. 15 Pareto optimal solutions of problem (19): (a) Solution A*; (b) Solution B*; (c) Solution $\mathrm{C}^{*} ;$ (d) Solution $\mathrm{D}^{*}$

Note that the arguments $\boldsymbol{A}, \boldsymbol{E}_{\mathrm{p}}$ and $\boldsymbol{x}_{\mathrm{p}}$ of order statistics in the right-hand side of Eq. (20) have been omitted for brevity. After solving problem (19), we select four solutions $A^{*}, B^{*}, C^{*}$ and $D^{*}$ among the Pareto optimal solutions which have the smallest objective values of $\sigma^{\max }, \hat{\sigma}_{2: 200}^{\max }$, $\hat{\sigma}_{50: 200}^{\max }$ and $\hat{\sigma}_{100: 200}^{\max }$, respectively. These solutions and the corresponding distribution of crosssectional areas are plotted in Figs. 15 and 16, respectively. Compared to the results obtained by solving problem (18), we can see that although solutions $\mathrm{A}^{*}, \mathrm{~B}^{*}, \mathrm{C}^{*}$ and $\mathrm{D}^{*}$ are not exactly the same as solutions A, B, C and D, respectively, they have similar shape and topology after removing 
thin elements with cross-sectional areas less than $0.0001 \mathrm{~m}^{2}$. Their distributions of cross-sectional areas are also close, demonstrating that multiobjective optimization problems (18) and (19) would lead to similar structures. We list the objective values and structural volume of solutions A*, B*, $\mathrm{C}^{*}$ and $\mathrm{D}^{*}$ before removing thin elements in Table 8, and the conclusion drawn from Table 7 can also apply for Table 8 .

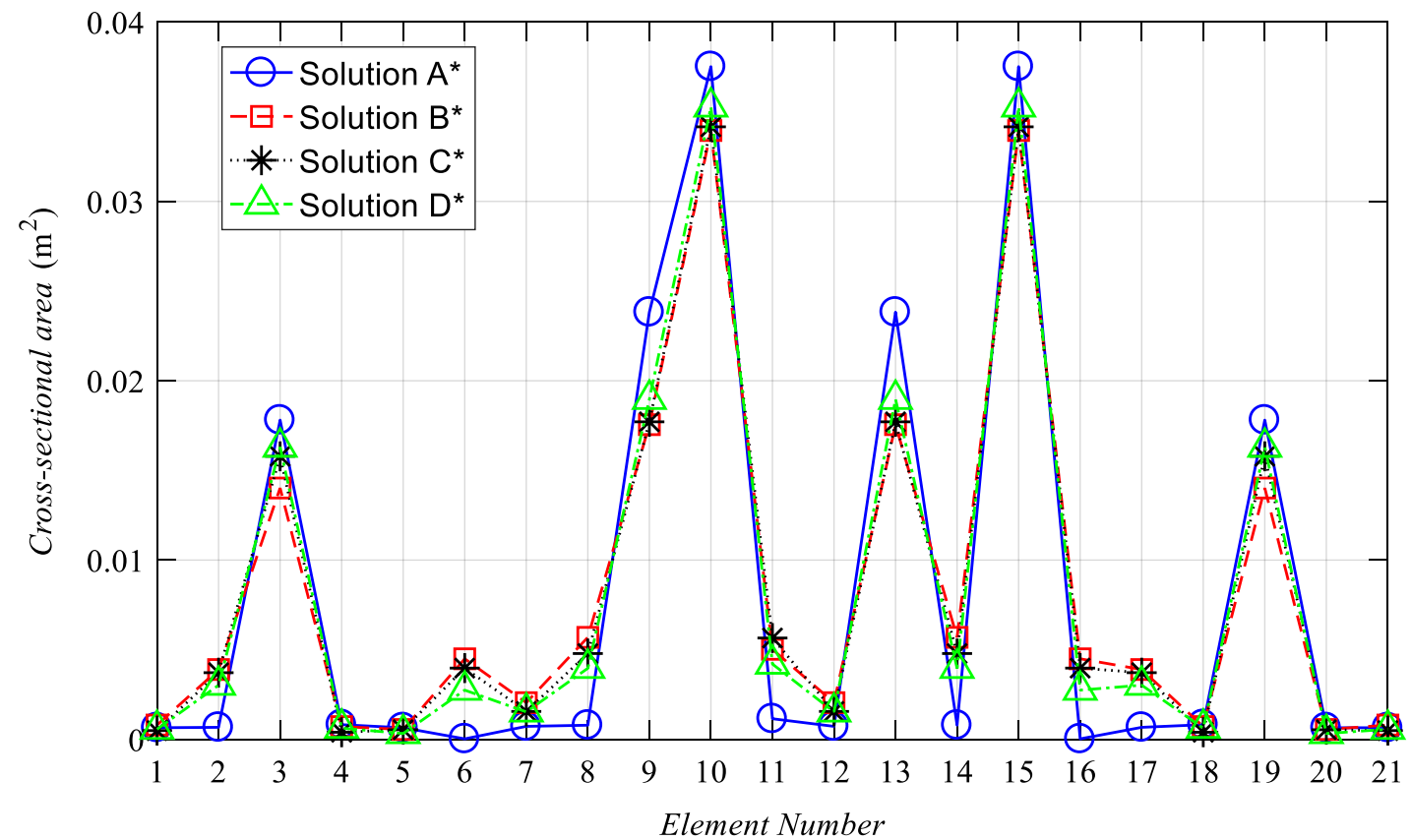

Fig. 16 Distribution of cross-sectional areas of solution $A^{*}, B^{*}, C^{*}$ and $D^{*}$ of problem (19) in Example 2

Table 8 Objective values and structural volume of solutions $A^{*}, B^{*}, C^{*}$ and $D^{*}$ of problem (19) in Example 2 before thin elements

\begin{tabular}{|c|c|c|c|c|c|}
\hline Solution & $\sigma^{\max }(\mathrm{Pa})$ & $\hat{\sigma}_{2: 200}^{\max }(\mathrm{Pa})$ & $\hat{\sigma}_{50: 200}^{\max }(\mathrm{Pa})$ & $\hat{\sigma}_{100: 200}^{\max }(\mathrm{Pa})$ & Volume $\left(\mathrm{m}^{3}\right)$ \\
\hline $\mathrm{A}^{*}$ & 3962.36 & 14762.3 & 9432.21 & 7155.88 & 0.2 \\
\hline $\mathrm{B}^{*}$ & 5150.72 & 6572.23 & 5925.88 & 5504.99 & 0.2 \\
\hline $\mathrm{C}^{*}$ & 4790.73 & 6820.37 & 5700.54 & 5255.23 & 0.2 \\
\hline $\mathrm{D}^{*}$ & 4580.95 & 6981.48 & 5842.58 & 5180.97 & 0.2 \\
\hline
\end{tabular}

Based on the solutions of problems (18) and (19), we further investigate the variations of solutions $\mathrm{A}, \mathrm{B}, \mathrm{C}, \mathrm{D}$ and $\mathrm{A}^{*}, \mathrm{~B}^{*}, \mathrm{C}^{*}, \mathrm{D}^{*}$, respectively. 2000 random seeds are used to generate 2000 groups of 200 samples for $\boldsymbol{E}_{\mathrm{p}}$ and $\boldsymbol{x}_{\mathrm{p}}$, and the mean values and standard deviations of the objective functions $\sigma^{\max }, \sigma_{1: 200}^{\max }, \sigma_{50: 200}^{\max }, \sigma_{100: 200}^{\max }$ and $\sigma^{\max }, \hat{\sigma}_{2: 200}^{\max }, \hat{\sigma}_{50: 200}^{\max }, \hat{\sigma}_{100: 200}^{\max }$ are listed in Tables 9 and 10, respectively. It can be observed from Table 9 that the standard deviation of $\sigma^{\max }$ 
for each solution is 0 , and the corresponding mean values are the same as those listed in Table 7. Besides, solutions A, B, C and D have the smallest mean value of objective functions $\sigma^{\max }, \sigma_{1: 200}^{\max }$, $\sigma_{50: 200}^{\max }$ and $\sigma_{100: 200}^{\max }$, respectively, which confirms the accuracy of optimization process. It is worth noting that solution $\mathrm{B}$ has the largest standard deviation of $\sigma_{1: 200}^{\max }$ among all the objective functions and at the same time has the smallest standard deviation of $\sigma_{1: 200}^{\max }$ among all the four solutions, and the standard deviation of $\sigma_{1: 200}^{\max }$ for solution B is larger than the standard deviations of $\sigma_{50: 200}^{\max }$ and $\sigma_{100: 200}^{\max }$ for solutions $\mathrm{C}$ and $\mathrm{D}$, respectively. This verifies that the extreme value corresponding to $k=1$ has more variation than the order statistics with larger values of $k$.

The same conclusion can be drawn from Table 10 for solutions $\mathrm{A}^{*}, \mathrm{~B}^{*}, \mathrm{C}^{*}$ and $\mathrm{D}^{*}$ and objective functions $\sigma^{\max }, \hat{\sigma}_{2: 200}^{\max }, \hat{\sigma}_{50: 200}^{\max }$ and $\hat{\sigma}_{100: 200}^{\max }$. Comparison between Tables 9 and 10 shows that the standard deviations of $\hat{\sigma}_{2: 200}^{\max }$ for solution $\mathrm{B}^{*}, \hat{\sigma}_{50: 200}^{\max }$ for solution $\mathrm{C}^{*}$ and $\hat{\sigma}_{100: 200}^{\max }$ for solution $\mathrm{D}^{*}$ are smaller than those of $\sigma_{1: 200}^{\max }$ for solution $\mathrm{B}, \sigma_{50: 200}^{\max }$ for solution $\mathrm{C}$ and $\hat{\sigma}_{100: 200}^{\max }$ for solution $\mathrm{D}$, respectively, indicating that the robust optimization using trimmed mean as objective functions will lead to solutions which are less sensitive to the uncertain parameters.

Table 9 Mean value $(\mathrm{Pa})$ and standard deviation $(\mathrm{Pa})$ of objective values of solutions $\mathrm{A}, \mathrm{B}, \mathrm{C}$ and $\mathrm{D}$ of problem (18) in Example 2

\begin{tabular}{|c|c|c|c|c|c|c|c|c|}
\hline Solution & \multicolumn{2}{|c|}{ A } & \multicolumn{2}{c|}{ B } & \multicolumn{3}{c|}{ C } & \multicolumn{2}{c|}{ D } \\
\hline Variation & Mean & $\begin{array}{c}\text { Std. } \\
\text { Dev }\end{array}$ & Mean & $\begin{array}{c}\text { Std. } \\
\text { Dev }\end{array}$ & Mean & $\begin{array}{c}\text { Std. } \\
\text { Dev }\end{array}$ & Mean & $\begin{array}{c}\text { Std. } \\
\text { Dev }\end{array}$ \\
\hline$\sigma^{\max }$ & 3949.13 & 0 & 5268.16 & 0 & 4780.91 & 0 & 4609.75 & 0 \\
\hline$\sigma_{1: 200}^{\max }$ & 15585.87 & 878.94 & 7018.64 & 206.94 & 7447.45 & 248.3 & 7495.61 & 251.74 \\
\hline$\sigma_{50: 200}^{\max }$ & 9344.63 & 288.23 & 5923.11 & 44.57 & 5757.67 & 86.42 & 5817.05 & 87.49 \\
\hline$\sigma_{100: 200}^{\max }$ & 7457.03 & 226.38 & 5646.81 & 32.03 & 5251.68 & 63.66 & 5218.24 & 58.97 \\
\hline
\end{tabular}

Table 10 Mean value $(\mathrm{Pa})$ and standard deviation $(\mathrm{Pa})$ of objective values of solutions $\mathrm{A}^{*}, \mathrm{~B}^{*}$, $\mathrm{C}^{*}$ and $\mathrm{D}^{*}$ of problem (19) in Example 2

\begin{tabular}{|c|c|c|c|c|c|c|c|c|}
\hline Solution & \multicolumn{2}{|c|}{$\mathrm{A}^{*}$} & \multicolumn{2}{c|}{$\mathrm{B}^{*}$} & \multicolumn{3}{c|}{$\mathrm{C}^{*}$} & \multicolumn{2}{c|}{$\mathrm{D}^{*}$} \\
\hline Variation & Mean & $\begin{array}{c}\text { Std. } \\
\text { Dev }\end{array}$ & Mean & $\begin{array}{c}\text { Std. } \\
\text { Dev }\end{array}$ & Mean & $\begin{array}{c}\text { Std. } \\
\text { Dev }\end{array}$ & Mean & $\begin{array}{c}\text { Std. } \\
\text { Dev }\end{array}$ \\
\hline$\sigma^{\max }$ & 3962.36 & 0 & 5150.72 & 0 & 4790.73 & 0 & 4580.94 & 0 \\
\hline$\hat{\sigma}_{2: 200}^{\max }$ & 14842.48 & 674.77 & 6877.83 & 150.88 & 7094.47 & 168.92 & 7320.2 & 184.88 \\
\hline$\hat{\sigma}_{50: 200}^{\max }$ & 9274.31 & 291.96 & 5841.14 & 50.08 & 5701.11 & 79.4 & 5812.24 & 88.97 \\
\hline$\hat{\sigma}_{100: 200}^{\max }$ & 7368.72 & 226.08 & 5532.99 & 34.3 & 5229.55 & 65.68 & 5206.35 & 53.4 \\
\hline
\end{tabular}


In order to compare the convergence properties of the optimization process, we solve problems (18) and (19), respectively, 10 times with different random seeds. From each trial we can obtain the sets of solutions $\mathrm{A}, \mathrm{B}, \mathrm{C}, \mathrm{D}$ and $\mathrm{A}^{*}, \mathrm{~B}^{*}, \mathrm{C}^{*}$ and $\mathrm{D}^{*}$ which have the smallest values of the corresponding four objective functions, respectively, among the Pareto optimal solutions. Then we calculate the mean value, standard deviation, maximum and minimum values of these solution sets as listed in Table 11. It can be observed that for $\sigma^{\max }$ the difference of the statistical information between the two solution sets is very small. This is because the optimal solution of problem (17) is added into the initial population of both problems (18) and (19). By contrast, the standard deviations of $\sigma_{1: 200}^{\max }, \sigma_{50: 200}^{\max }$ and $\sigma_{100: 200}^{\max }$ are larger than those of $\hat{\sigma}_{2: 200}^{\max }, \hat{\sigma}_{50: 200}^{\max }$ and $\hat{\sigma}_{100: 200}^{\max }$, respectively. This is mainly because the trimmed mean is less sensitive to the outliers in the samples and can be referred as a robust estimator of quantile structural response. Therefore, the multiobjective optimization problem (19) is more stable than those of problem (18) due to the smoothness of the objective functions during the optimization process.

Table 11. Statistical information of problems (18) and (19) in Example 2

\begin{tabular}{|c|c|c|c|c|c|c|c|c|}
\hline Solution set & \multicolumn{4}{|c|}{ Problem (18) } & \multicolumn{4}{c|}{ Problem (19) } \\
\hline Objective & $\sigma^{\max }$ & $\sigma_{1: 200}^{\max }$ & $\sigma_{50: 200}^{\max }$ & $\sigma_{100: 200}^{\max }$ & $\sigma^{\max }$ & $\hat{\sigma}_{2: 200}^{\max }$ & $\hat{\sigma}_{50: 200}^{\max }$ & $\hat{\sigma}_{100: 200}^{\max }$ \\
\hline Max. $(\mathrm{Pa})$ & 3971.81 & 7255.42 & 5813.48 & 5219.33 & 3969.66 & 7056.81 & 5815.25 & 5203.84 \\
\hline Min. $(\mathrm{Pa})$ & 3949.13 & 6618.92 & 5555.50 & 5039.94 & 3948.07 & 6565.64 & 5611.88 & 5067.77 \\
\hline Mean $(\mathrm{Pa})$ & 3965.95 & 6904.60 & 5696.22 & 5144.06 & 3965.49 & 6742.10 & 5699.17 & 5138.22 \\
\hline Std. Dev. $(\mathrm{Pa})$ & 5.86 & 227.70 & 70.56 & 50.37 & 5.67 & 162.50 & 60.67 & 46.38 \\
\hline
\end{tabular}

\subsection{Example 3}

The last example investigates a cantilever beam with a $3 \times 2$ grid, and its geometry information is shown in Fig. 17(a). The structure is pin-supported at nodes 1, 2 and 3, and a downward vertical load $P=100 \mathrm{~N}$ is applied at node 11 . Based on symmetry of the structure, we select the design variables as $\boldsymbol{x}=\left(x_{4}, x_{5}, x_{7}, x_{8}, x_{10}, y_{4}, y_{7}, y_{10}\right)$ and $\boldsymbol{A}=\left(A_{1}, A_{2}, \ldots A_{15}\right)$, which represent the $x$ coordinates of nodes $4,5,7,8$ and 10 and $y$-coordinates of nodes 4,7 and 10, and the crosssectional areas of elements 1 to 15. The parameter values are listed in Table 3. Because in shape optimization of frame structure the stiffness matrix may become singular due to the melting nodes (Achtziger 2007; Ohsaki and Hayashi 2017; Shen and Ohsaki 2020), we add box constraints to nodes $4,5,7,8$ and 10 as

$$
\begin{aligned}
& 0.1 \leq x_{4}, x_{5} \leq 1.45 ; \quad 1.55 \leq x_{7} \leq 2.45 ; \quad 1.55 \leq x_{8} \leq 3 \\
& 2.55 \leq x_{10} \leq 3 ; \quad 0 \leq y_{4}, y_{7}, y_{10} \leq 0.9
\end{aligned}
$$

and indicate them using the dotted red lines in Fig. 17(b). 

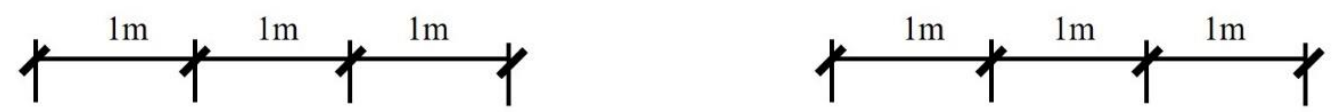

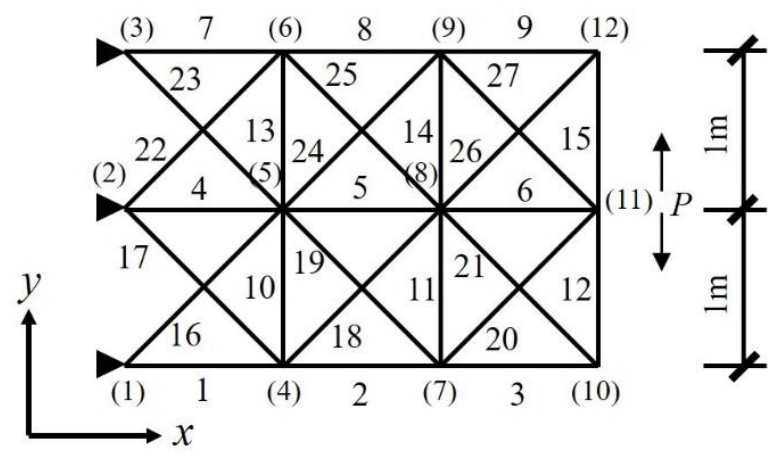

(a)

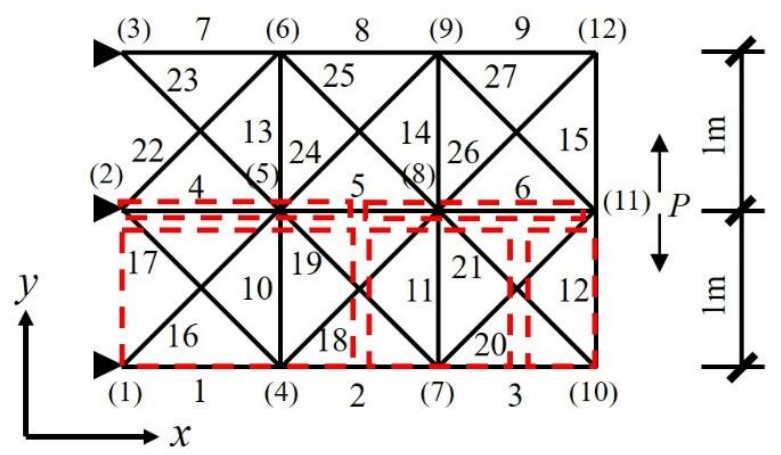

(b)

Fig. 17 (a) Initial ground structure of Example 3; (b) Limitation on the moving range of nodes

Assuming that the uncertain parameters are the Young's modulus of each element and the $x$ and $y$-coordinates of all the nodes except 1,2,3 and 11, we formulate the single objective optimization problem to minimize the maximum as

$$
\begin{array}{ll}
\text { Minimize } & \sigma^{\max }=\max _{i=1,2, \ldots, 15} \sigma_{i}^{\max }\left(\boldsymbol{A}, \boldsymbol{x}, \overline{\boldsymbol{E}}_{\mathrm{p}}\right) \\
\text { subject to } & V(\boldsymbol{A}, \boldsymbol{x}) \leq V_{\mathrm{U}} ; A_{\mathrm{L}} \leq A_{l} \leq A_{\mathrm{U}}, \quad l=1,2, \cdots 15 \\
& 0.1 \leq x_{4}, x_{5} \leq 1.45 ; \quad 1.55 \leq x_{7} \leq 2.45 ; \quad 1.55 \leq x_{8} \leq 3 \\
& 2.55 \leq x_{10} \leq 3 ; \quad 0 \leq y_{4}, y_{7}, y_{10} \leq 0.9
\end{array}
$$

The optimal solution of problem (22) is presented in Fig. 18, which is added to the initial population of the following multiobjective optimization problem:

Minimize $\sigma^{\max }\left(\boldsymbol{A}, \overline{\boldsymbol{E}}_{\mathrm{p}}, \overline{\boldsymbol{x}}_{\mathrm{p}}\right), \sigma_{1: 200}^{\max }\left(\boldsymbol{A}, \boldsymbol{E}_{\mathrm{p}}, \boldsymbol{x}_{\mathrm{p}}\right), \sigma_{50: 200}^{\max }\left(\boldsymbol{A}, \boldsymbol{E}_{\mathrm{p}}, \boldsymbol{x}_{\mathrm{p}}\right), \sigma_{100: 200}^{\max }\left(\boldsymbol{A}, \boldsymbol{E}_{\mathrm{p}}, \boldsymbol{x}_{\mathrm{p}}\right)$

subject to $V(\boldsymbol{A}) \leq V_{\mathrm{U}} ; A_{\mathrm{L}} \leq A_{l} \leq A_{\mathrm{U}}, \quad l=1,2, \ldots, 15$ 


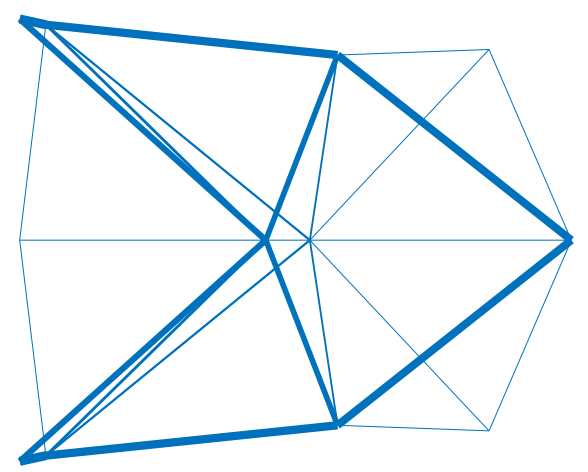

Fig. 18 Optimal solution of problem (22)

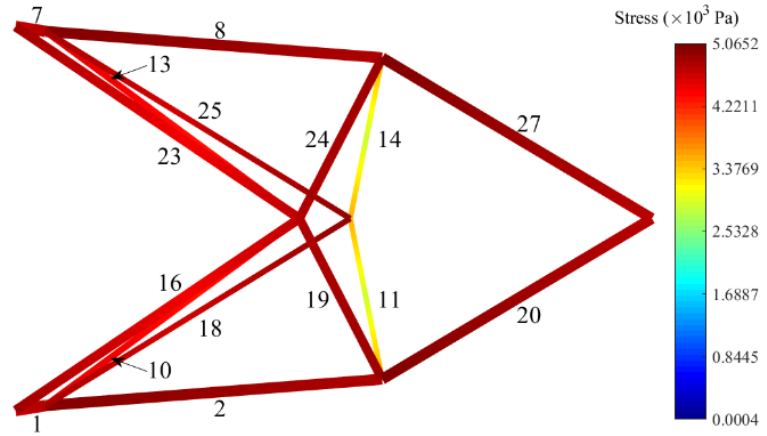

(a)

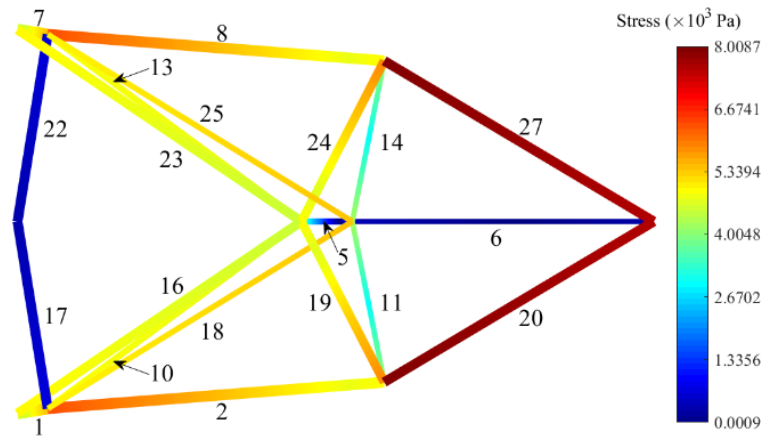

(c)

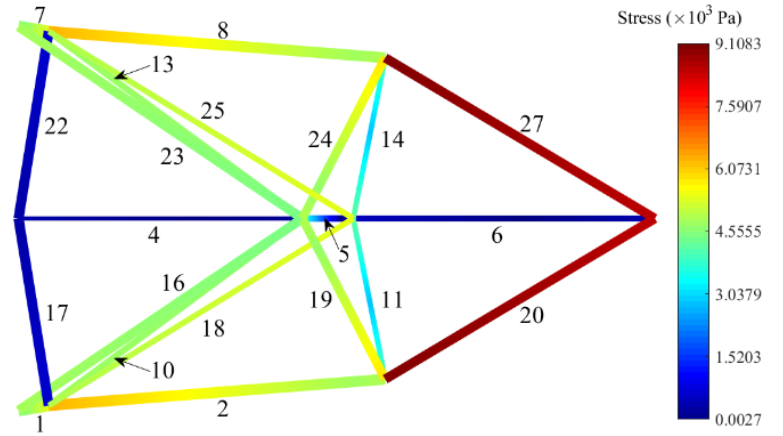

(b)

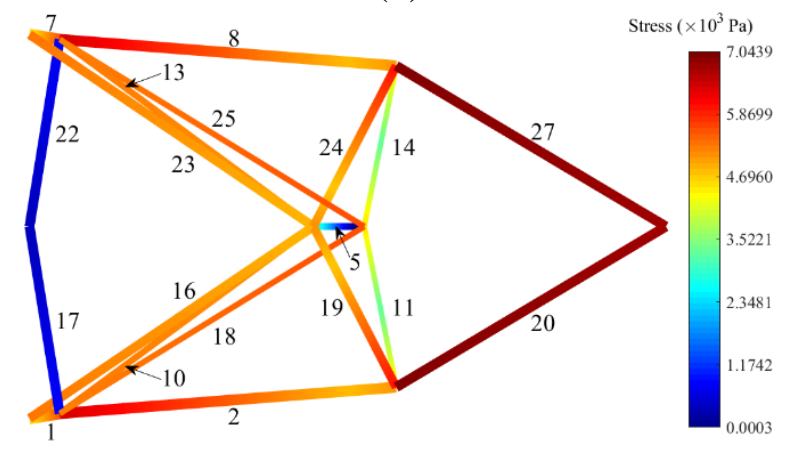

(d)

Fig. 19 Pareto optimal solutions of problem (23): (a) Solution A; (b) Solution B; (c) Solution C; (d) Solution D

We obtain four solutions A, B, C and D from the 70 Pareto solutions of problem (23) which have the smallest objective values of $\sigma^{\max }, \sigma_{1: 200}^{\max }, \sigma_{50: 200}^{\max }$ and $\sigma_{100: 200}^{\max }$, respectively, and the total computational costs for solving problems (22) and (23) are listed in Table 4. The four solutions are shown in Fig. 19 after removal of thin elements whose cross-sectional areas are less than 0.0001 $\mathrm{m}^{2}$, and their distributions of maximum edge stress are also displayed in Fig. 19. 
As seen from Fig. 19, structures with different robustness levels can be obtained by solving problem (23) and the number of existing elements in the final topoloy decreases from solution B to solution $\mathrm{D}$. This is mainly because solution $\mathrm{B}$ has the minimum objective value of $\sigma_{1: 200}^{\max }$ among the Pareto solutions, resulting in more necessary elements to reduce the maximum structural stress under uncertainty, and for the objective functions $\sigma_{50: 200}^{\max }$ and $\sigma_{100: 200}^{\max }$ with lower robustness, fewer elements are retained in solutions $\mathrm{C}$ and D. It is interesting to note that solution D has the similar structural topology as solution A except for the existence of element 5, indicating that the optimal solution for median response is close to the solution without uncertainty.

Table 12 Objective values and structural volume of solutions A, B, C and D of problem (23) in Example 3 before removing thin elements

\begin{tabular}{|c|c|c|c|c|c|}
\hline Solution & $\sigma^{\max }(\mathrm{Pa})$ & $\sigma_{1: 200}^{\max }(\mathrm{Pa})$ & $\sigma_{50: 200}^{\max }(\mathrm{Pa})$ & $\sigma_{100: 200}^{\max }(\mathrm{Pa})$ & Volume $\left(\mathrm{m}^{3}\right)$ \\
\hline $\mathrm{A}$ & 5065.19 & 28101.49 & 19310.89 & 16307.84 & 0.2 \\
\hline $\mathrm{B}$ & 9108.34 & 18531.84 & 11578.97 & 10660.53 & 0.2 \\
\hline $\mathrm{C}$ & 8008.69 & 19210.26 & 11245.16 & 9720.72 & 0.2 \\
\hline $\mathrm{D}$ & 7043.87 & 19779.94 & 11633.44 & 9333.17 & 0.2 \\
\hline
\end{tabular}

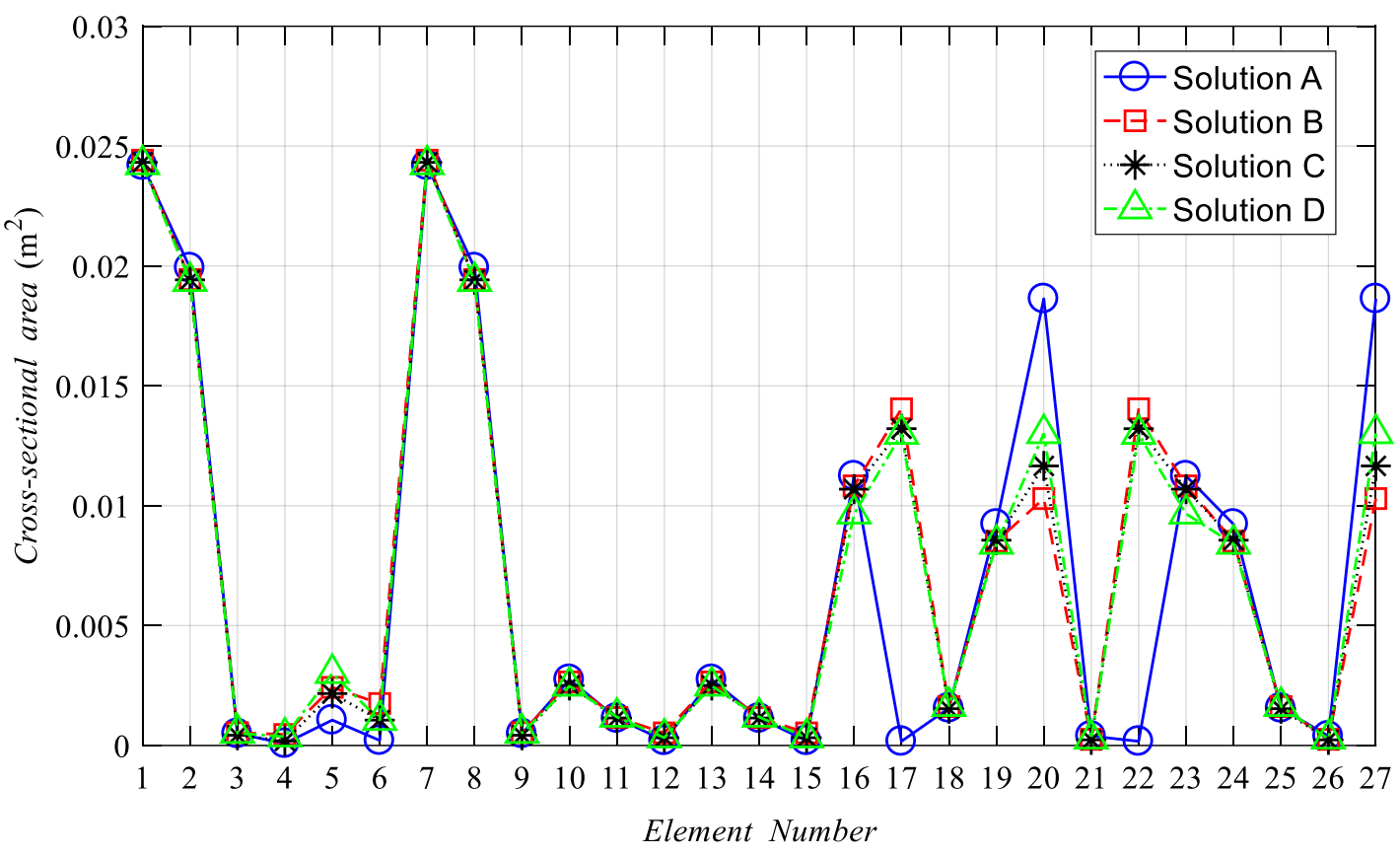

Fig. 20 Distribution of cross-sectional areas of solutions A, B, C and D of problem (23) in Example 3 
Table 12 lists the objective values and the structural volume of solutions A, B, C and D. Distribution of cross-sectional areas is plotted in Fig. 20. It can be observed that different robustness levels lead to different distributions of cross-sectional areas. Moreover, although the decrease from $\sigma_{50: 200}^{\max }$ to $\sigma_{100: 200}^{\max }$ is smaller than the decrease from $\sigma_{1: 200}^{\max }$ to $\sigma_{50: 200}^{\max }$ for solutions $\mathrm{B}, \mathrm{C}$ and $\mathrm{D}$, the minimum values of the three objective functions $\sigma_{1: 200}^{\max }, \sigma_{50: 200}^{\max }$ and $\sigma_{100: 200}^{\max }$ increase with the decrease of order $k$, which shows a trade-off relationship between the robustness level and the maximum stress in the solutions with same structural volume. Hence, the designers can choose the structure from Pareto optimal solutions that meets the desired value of maximum structural stress and robustness level.

\section{Conclusion}

A worst-case design approach is proposed for robust optimization of plane frame structure. To avoid large computational cost, the worst value of structural response is represented by the quantile response. Order statistics and trimmed mean are used for approximation of the quantile response and defining the robustness level based on the theory of distribution free one-side tolerance interval. It has been shown by Ohsaki et al. (2019) that the order $k$ can be used as robustness indicator if the sample size and confidence level are specified. We formulate a new multiobjective robust shape and topology optimization for plane frame structure using order statistics and trimmed means of the structural response with various orders to simultaneously obtain solutions with different robustness level.

The optimization procedure mainly contains two stages, the first stage is to solve a single objective shape and topology optimization problem and the second stage is to solve a multiobjective optimization problem where the order statistics or trimmed means of the structural response are served as multiple objectives, together with the one without considering uncertainty. In the first stage, the design variables are selected as nodal locations and cross-sectional areas, and the single objective optimization problem is solved with the nominal value of uncertain parameters. Based on the optimal solution of the first stage, we select only cross-sectional areas as design variables, and generate variations in uncertain parameters with required sample size for multiobjective optimization problem. After optimization in the second stage, the elements with small cross-sectional areas are removed to obtain the final topologies of the Pareto optimal solutions. Problems in both stages are solved by GAs, and LHS method is introduced to generate the initial population with diverse solutions.

Three numerical examples are presented to investigate and illustrate the effectiveness of the proposed method, and the values of order $k$ of order statistics and trimmed mean are selected in accordance with the maximum, quartile and median structural response. The uncertainty is considered in Young's modulus and nodal locations with approximately 10\% variation from their nominal values. It has been shown that some elements, which have little contribution to the reduce of structural stress and can be removed from the optimal solution without uncertainty, have increased their cross-sectional areas after robust optimization and are helpful of reducing the 
structural response under uncertainty. Thus, existence of uncertainty leads to a different topology of optimal solution.

Two multiobjective optimization problems are solved minimizing order statistics and trimmed means, respectively, and the variability of the optimal solutions with different robustness level is also discussed with 2000 random sets of uncertain parameters. Results show that the solutions obtained by using trimmed means have smaller standard deviations than those obtained by using simple order statics. In addition, comparison of results obtained from 10 different random seeds shows that multiobjective optimization procedure with trimmed means is more stable than the one with order statistics, indicating that trimmed means is less sensitive to the outliers in the samples and referred to a better indicator of the structure robustness.

However, as discussed by Sigmund (2011), when the sensitivity analysis is available for both objective and constraint functions, one should solve the optimization problem by a gradient-based method rather than a non-gradient method due to its computational efficiency. Further investigation and research are needed on how to derive the gradients for multiple objectives under uncertainty and how they influence the accuracy and efficiency of the proposed method. Moreover, with regard to the application to real engineering problem, surrogate model and other truncated approximation could be used to replace the real finite element analysis when it is time-consuming.

\section{Replication of results}

All the results in this research are obtained using homemade MATLAB codes. The source code and the research data can be available from the corresponding author with reasonable request.

\section{Compliance with ethical standards}

Conflict of interest: The authors declare that they have no conflict of interest.

\section{Acknowledgements}

This research is partially supported by China Scholarship Council (File No. 201806050114).

\section{Reference}

Achtziger W (2007) On simultaneous optimization of truss geometry and topology. Struct Multidiscip Optim 33:285-304. doi: 10.1007/s00158-006-0092-0

Asadpoure A, Tootkaboni M, Guest JK (2011) Robust topology optimization of structures with uncertainties in stiffness - Application to truss structures. Comput Struct 89:1131-1141. doi: 10.1016/j.compstruc.2010.11.004

Au FTK, Cheng YS, Tham LG, Zeng GW (2003) Robust design of structures using convex models. Comput Struct 81:2611-2619. doi: 10.1016/S0045-7949(03)00322-5 
Avérous J, Genest C, Kochar SC (2005) On the dependence structure of order statistics. J Multivar Anal 94:159-171. doi: 10.1016/j.jmva.2004.03.004

Ben-Tal A, Laurent El Ghaoui, Nemirovski A (2009) Robust Optimization. Princeton University Press

Ben-Tal A, Nemirovski A (2002) Robust optimization - Methodology and applications. Math Program Ser B 92:453-480. doi: 10.1007/s101070100286

Boland PJ, Hollander M, Joag-Dev K, Kochar S (1996) Bivariate dependence properties of order statistics. J Multivar Anal 56:75-89. doi: 10.1006/jmva.1996.0005

Cheng G, Xu L, Jiang L (2006) A sequential approximate programming strategy for reliabilitybased structural optimization. Comput Struct 84:1353-1367. doi:

10.1016/j.compstruc.2006.03.006

Choi S-K, Grandhi R V., Canfield RA (2007) Reliability-based Structural Design. Springer, London

Doltsinis I, Kang Z (2004) Robust design of structures using optimization methods. Comput Methods Appl Mech Eng 193:2221-2237. doi: 10.1016/j.cma.2003.12.055

Du L, Choi KK (2008) An inverse analysis method for design optimization with both statistical and fuzzy uncertainties. Struct Multidiscip Optim 37:107-119. doi: 10.1007/s00158-0070225-0

Du X, Chen W (2004) Sequential Optimization and Reliability Assessment Method for Efficient Probabilistic Design. J Mech Des 126:225. doi: 10.1115/1.1649968

Du X, Chen W (2000) Towards a better understanding of modeling feasibility robustness in engineering design. J Mech Des Trans ASME 122:385-394. doi: 10.1115/1.1290247

Dunning PD, Kim HA (2013) Robust Topology Optimization: Minimization of Expected and Variance of Compliance. AIAA J 51:2656-2664. doi: 10.2514/1.J052183

Elishakoff I, Ohsaki M (2010) Optimization and Anti-optimization of Structures Under Uncertainty. World Scientific, London

Enevoldsen I, Sorensen J. (1994) Reliability-based optimization in structural engineering. Struct Saf 15:169-196

Frangopol DM (1985) Structural optimization using reliability concepts. J Struct Eng 111:22882301. doi: 10.1061/(ASCE)0733-9445(1985)111:11(2288)

Guest JK, Igusa T (2008) Structural optimization under uncertain loads and nodal locations. Comput Methods Appl Mech Eng 198:116-124. doi: 10.1016/j.cma.2008.04.009

Guo X, Bai W, Zhang W, Gao X (2009) Confidence structural robust design and optimization under stiffness and load uncertainties. Comput Methods Appl Mech Eng 198:3378-3399. doi: 10.1016/j.cma.2009.06.018

Izui K, Yamada T, Nishiwaki S, Tanaka K (2015) Multiobjective optimization using an aggregative gradient-based method. Struct Multidiscip Optim 51:173-182. doi: 


\section{$10.1007 / \mathrm{s} 00158-014-1125-8$}

Jalalpour M, Guest JK, Igusa T (2013) Reliability-based topology optimization of trusses with stochastic stiffness. Struct Saf 43:41-49. doi: 10.1016/j.strusafe.2013.02.003

Jekel CF, Haftka RT (2020) Risk Allocation for Design Optimization with Unidentified Statistical Distributions. 1-9. doi: 10.2514/6.2020-0415

Kanno Y, Takewaki I (2006a) Sequential semidefinite program for maximum robustness design of structures under load uncertainty. J Optim Theory Appl 130:265-287. doi: $10.1007 / \mathrm{s} 10957-006-9102-\mathrm{Z}$

Kanno Y, Takewaki I (2006b) Confidence ellipsoids for static response of trusses with load and structural uncertainties. Comput Methods Appl Mech Eng 196:393-403. doi: 10.1016/j.cma.2006.04.007

Kharmanda G, Olhoff N, Mohamed A, Lemaire M (2004) Reliability-based topology optimization. Struct Multidiscip Optim 26:295-307. doi: 10.1007/s00158-003-0322-7

Kogiso N, Yang Y-S, Kim B-J, Lee J-O (2012) Modified Single-Loop-Single-Vector Method for Efficient Reliability-Based Design Optimization. J Adv Mech Des Syst Manuf 6:12061221. doi: 10.1299/jamdsm.6.1206

Lee I, Choi KK, Gorsich D (2010) System reliability-based design optimization using the MPPbased dimension reduction method. Struct Multidiscip Optim 41:823-839. doi: 10.1007/s00158-009-0459-0

Lee KH, Park GJ (2001) Robust optimization considering tolerances of design variables. Comput Struct 79:77-86. doi: 10.1016/S0045-7949(00)00117-6

Lombardi M, Haftka RT (1998) Anti-optimization technique for structural design under load uncertainties. Comput Methods Appl Mech Eng 157:19-31. doi: 10.1016/S00457825(97)00148-5

Mathworks (2018) Optimzation Toolbox User's Guide R2018a

Maxwell JC (1870) On Reciprocal Figures, Frames, and Diagrams of Forces. Trans R Soc Edinburgh 161-207. doi: 10.1017/S0080456800026351

Michell AGM (1904) The Limits of Economy of 1llaterial in Frame-structures . Philos Mag Ser 6 8:589-597. doi: 10.1080/14786440409463229

Mogami K, Nishiwaki S, Izui K, et al (2006) Reliability-based structural optimization of frame structures for multiple failure criteria using topology optimization techniques. Struct Multidiscip Optim 32:299-311. doi: 10.1007/s00158-006-0039-5

Navarro J, Balakrishnan N (2010) Study of some measures of dependence between order statistics and systems. J Multivar Anal 101:52-67. doi: 10.1016/j.jmva.2009.04.016

Ohsaki M, Hayashi K (2017) Force density method for simultaneous optimization of geometry and topology of trusses. Struct Multidiscip Optim 56:1157-1168. doi: 10.1007/s00158-017$1710-8$ 
Ohsaki M, Katsura M (2012) A random sampling approach to worst-case design of structures. Struct Multidiscip Optim 46:27-39. doi: 10.1007/s00158-011-0752-6

Ohsaki M, Yamakawa M, Fan W, Li Z (2019) An order statistics approach to multiobjective structural optimization considering robustness and confidence of responses. Mech Res Commun 97:33-38. doi: 10.1016/j.mechrescom.2019.04.003

Picheny V, Kim NH, Haftka RT (2010) Application of bootstrap method in conservative estimation of reliability with limited samples. Struct Multidiscip Optim 41:205-217. doi: $10.1007 / \mathrm{s} 00158-009-0419-8$

Prescott P, Arnold BC, Balakrishnan N, Nagaraja HN (1993) A First Course in Order Statistics. Society for Industrial and Applied Mathematics

Qu X, Haftka RT (2004) Reliability-based design optimization using probabilistic sufficiency factor. Struct Multidiscip Optim 27:314-325. doi: 10.1007/s00158-004-0390-3

Rajan SD (1995) Sizing, shape, and togology design optimization of trusses using genetic algorithm. J Struct Eng 121:1480-1487. doi: 10.1007/978-3-319-93025-1_4

Richardson JN, Filomeno Coelho R, Adriaenssens S (2015) Robust topology optimization of truss structures with random loading and material properties: A multiobjective perspective. Comput Struct 154:41-47. doi: 10.1016/j.compstruc.2015.03.011

Shen W, Ohsaki M (2020) Geometry and topology optimization of plane frames for compliance minimization using force density method for geometry model. Eng Comput. doi: 10.1007/s00366-019-00923-w

Sigmund O (2011) On the usefulness of non-gradient approaches in topology optimization. Struct Multidiscip Optim 43:589-596. doi: 10.1007/s00158-011-0638-7

Takezawa A, Nii S, Kitamura M, Kogiso N (2011) Topology optimization for worst load conditions based on the eigenvalue analysis of an aggregated linear system. Comput Methods Appl Mech Eng 200:2268-2281. doi: 10.1016/j.cma.2011.03.008

Tootkaboni M, Asadpoure A, Guest JK (2012) Topology optimization of continuum structures under uncertainty - A Polynomial Chaos approach. Comput Methods Appl Mech Eng 201204:263-275. doi: 10.1016/j.cma.2011.09.009

Venter G, Haftka RT (2010) Constrained particle swarm optimization using a bi-objective formulation. Struct Multidiscip Optim 40:65-76. doi: 10.1007/s00158-009-0380-6

Wang L, Cai Y, Liu D (2018) Multiscale reliability-based topology optimization methodology for truss-like microstructures with unknown-but-bounded uncertainties. Comput Methods Appl Mech Eng 339:358-388. doi: 10.1016/j.cma.2018.05.003

Wang L, Liang J, Liu D, Chen W (2019) A novel reliability-based topology optimization framework for the concurrent design of solid and truss-like material structures with unknown-but-bounded uncertainties. Int J Numer Methods Eng 1-22. doi: 10.1002/nme.6048

Yamakawa M, Ohsaki M (2016) Robust design optimization considering parameter variation of 
seismic characteristics using order statistics. Jounal Struct Eng (In Japanese) 62B:381-388

Zavala GR, Nebro AJ, Luna F, Coello Coello CA (2014) A survey of multi-objective metaheuristics applied to structural optimization. Struct Multidiscip Optim 49:537-558. doi: 10.1007/s00158-013-0996-4 